\title{
ON RIGIDITY, LIMIT SETS, AND END INVARIANTS OF HYPERBOLIC 3-MANIFOLDS
}

\author{
YAIR N. MINSKY
}

\section{INTRODUCTION}

A central feature of the study of Kleinian groups is the interplay between 3-dimensional hyperbolic geometry and conformal dynamics. A Kleinian group $\Gamma$ is a discrete subgroup of $\operatorname{PSL}_{2}(\mathbf{C})$, which acts continuously on the union $\mathbf{H}^{3} \cup \hat{\mathbf{C}}$ of the hyperbolic 3-space with its natural boundary, the Riemann sphere $\hat{\mathbf{C}}=\mathbf{C} \cup\{\infty\}$. In the interior $\Gamma$ acts by hyperbolic isometries, and on the boundary by conformal homeomorphisms.

Thus one obtains connections between the geometry of the hyperbolic 3manifolds (or more generally, orbifolds) obtained as quotients $\mathbf{H}^{3} / \Gamma$, and the conformal dynamics of the action of $\Gamma$ on $\hat{\mathbf{C}}$. The natural questions of classification, structure, and deformation of these systems have been fruitfully approached from both of these points of view, and their interaction provides a great deal of structure.

This paper studies what is initially a question of classification. A hyperbolic 3-manifold $N$ of infinite volume has ends, which we think of as the connected components of the complement in $N$ of a compact submanifold which is homotopy-equivalent to $N$ (see section 2). If $N$ is "geometrically tame", the geometry of its ends can be approximately characterized by what we call end invariants (see sections 2 and 9). If an end is "geometrically finite" its invariant is a Riemann surface, which arises as the quotient of a component of the domain of discontinuity of the action of the associated Kleinian group on $\hat{\mathbf{C}}$. If an end is "simply degenerate", the invariant is a geodesic lamination (which can be thought of as an infinite curve system on a surface). These latter invariants, and the associated notions of simply degenerate ends and geometrically tame manifolds, were introduced by Thurston [Thu82a], who made the following conjecture (see also [Thu82b, Abi88]):

Received by the editors February 8, 1993.

1991 Mathematics Subject Classification. Primary 30F40, 57M50, 57M60; Secondary 30F60, 30 C62.

Research supported in part by an NSF postdoctoral fellowship. 
Conjecture 1.1 (Ending lamination conjecture) (Thurston). A hyperbolic 3-manifold with finitely generated fundamental group is uniquely determined by its topological type, and its list of end invariants.

We shall prove this conjecture in a special case, and apply the techniques of the proof to obtain some further results about the structure and deformations of some Kleinian groups. In particular we will try to clarify somewhat the connection of the ending laminations, which are "internal" hyperbolic objects, to the dynamics of the action of the group on $\hat{\mathbf{C}}$.

Let $N$ be a complete hyperbolic 3-manifold with finitely generated fundamental group $\Gamma \subset \mathrm{PSL}_{2}(\mathbf{C})$. In this paper we shall consider $N$ (or equivalently $\Gamma)$ which satisfies the following conditions:

C1. $\Gamma \neq A * B$ unless $A$ or $B$ is trivial.

C2. There is a lower bound $\epsilon>0$ on the injectivity radius at every point in $N$.

Condition $\mathrm{C} 1$ ensures, by a theorem of Bonahon [Bon86], that $N$ is geometrically tame. The question of geometric tameness is still open for hyperbolic manifolds not satisfying this condition. Canary [Can93] has shown that it is equivalent to the question of whether $N$ is homeomorphic to the interior of a compact manifold. In the case of such manifolds it seems possible that some of the techniques of this paper would apply, though the analysis has not been carried out.

Condition $\mathrm{C} 2$ allows some fairly strong use of compactness theorems in this paper, and in its predecessor [Min93]. Recall that the injectivity radius $\operatorname{inj}_{N}(x)$ for $x \in N$ is the supremum of radii of embedded balls about $x$.

Our main theorem is the following:

Theorem A (Ending lamination theorem). Let $N_{1}$ and $N_{2}$ be two homeomorphic hyperbolic 3-manifolds satisfying $\mathrm{C1}-2$. If the end invariants of corresponding ends of $N_{1}$ and $N_{2}$ are equal, then the homeomorphism is homotopic to an isometry.

Before we discuss this result and its corollaries, let us recall briefly the recent history of this aspect of Kleinian groups.

Theorem A can be seen as a "rigidity" result, and in fact is based on a wellknown family of such results. The first of these is the Mostow rigidity theorem [Mos68, Mos73], which states that two isomorphic Kleinian groups with compact quotients are in fact conjugate in $\operatorname{Isom}\left(\mathbf{H}^{3}\right)$. The isomorphism, together with the compactness of the quotient, gives rise to a quasi-isometry of $\mathbf{H}^{3}$ that conjugates the actions of the groups, and extends to a quasiconformal conjugacy of their actions on $\hat{\mathbf{C}}$. An ergodicity property of the group actions on their limit sets (which are all of $\hat{\mathbf{C}}$ in this case) then implies that the conjugacy is in fact conformal, and hence an isometry in $\mathbf{H}^{3}$. Mostow's theorem extends to groups with finite-volume quotients, by work of Prasad [Pra73]. 
The second classical example is the classification of geometrically finite groups by Bers, Kra, Marden, Maskit, et al. Such groups have quotients of infinite volume, but the geometry is controlled by a finite-volume region called the convex core. Such a group acts discontinuously on a non-empty domain $\Omega \subset \hat{\mathbf{C}}$ (the complement of its limit set), and the quotient of $\Omega$ is a finite collection of Riemann surfaces of finite type, which are the end invariants for the group. Two isomorphic geometrically finite groups are conjugate in $\operatorname{Isom}\left(\mathbf{H}^{3}\right)$ if these quotient Riemann surfaces are conformally equivalent. In other words, there are no deformations of such a group which are not reflected in deformations of the end invariants.

Finally there is Sullivan's rigidity theorem (see [Sul81] and section 6). This theorem states that two finitely generated Kleinian groups $\Gamma_{1}$ and $\Gamma_{2}$ are conjugate in $\operatorname{Isom}\left(\mathbf{H}^{3}\right)$ if their actions on $\hat{\mathbf{C}}$ are conjugate by a quasiconformal homeomorphism, which in addition is actually conformal on the domains of discontinuity. In the case of geometrically finite groups with infinite-volume quotients, this theorem reiterates the fact that the conformal boundary at infinity controls the geometry of the group. For geometrically infinite groups, the theorem shows that the conformal boundary still controls the quasiconformal deformations.

The question arises of whether the condition of quasiconformality of the conjugating homeomorphism is necessary in Sullivan's theorem, or whether a topological conjugacy suffices. The connection between this question and the ending lamination conjecture is that one expects two topologically conjugate Kleinian groups to have the same ending laminations for their degenerate ends-indeed the ending laminations were originally introduced to characterize the topological structure of the action of a Kleinian group on the sphere. We can prove the following, in the case of surface groups:

Theorem $\mathbf{E}$ (Topological rigidity). Let $\Gamma_{1}$ and $\Gamma_{2}$ be two Kleinian groups, isomorphic to a closed surface group $\pi_{1}(S)$, and satisfying C2. Suppose there is a homeomorphism $\mathscr{F}: \hat{\mathbf{C}} \rightarrow \hat{\mathbf{C}}$ such that

$$
\Gamma_{2}=\mathscr{F} \circ \Gamma_{1} \circ \mathscr{F}^{-1}
$$

(as groups acting on the sphere), and $\mathscr{F}$ is conformal on the domain of discontinuity of $\Gamma_{1}$. Then $\mathscr{F}$ is a Möbius transformation.

Remark. Ohshika [Ohs] has recently shown how to apply Theorems A and E to obtain a generalization of Theorem $\mathrm{E}$ to all groups satisfying $\mathrm{C} 1$ and $\mathrm{C} 2$.

Theorem $\mathrm{E}$ follows from a result that makes more precise the connection of the ending lamination to the topological structure of the action of a Kleinian group on its limit set. This is Theorem D, whose full statement appears in section 7 , and which may be briefly described as follows:

Theorem D (Topology of the action). Let $\rho_{0}: \pi_{1}(S) \rightarrow \mathrm{PSL}_{2}(\mathbf{C})$ be a Fuchsian representation of a closed surface group, and let $\rho: \pi_{1}(S) \rightarrow \mathrm{PSL}_{2}(\mathbf{C})$ be any 
discrete, faithful representation whose image satisfies $\mathrm{C} 2$. Then there is a continuous map $\pi: \hat{\mathbf{C}} \rightarrow \hat{\mathbf{C}}$ which takes the action of $\rho_{0}\left(\pi_{1}(S)\right)$ to that of $\rho\left(\pi_{1}(S)\right)$. This map collapses certain closed subsets of $\hat{\mathbf{C}}$ to points, and is determined up to isotopy by the end invariants of $\rho$.

This theorem extends a result originally proved by Cannon and Thurston [CT89] in the case of groups arising from a limiting construction of Thurston using pseudo-Anosov homeomorphisms of $S$ (in particular their result applies to groups of fibers of closed hyperbolic 3-manifolds fibering over the circle). As a consequence of Theorem $\mathrm{D}$ we also obtain the extension to our setting of another theorem of Cannon and Thurston:

Theorem B (Peano curves). Let $\Gamma=\rho\left(\pi_{1}(S)\right)$ be a Kleinian group satisfying $\mathrm{C} 2$, where $S$ is a closed surface, and let $\rho_{0}$ be a Fuchsian representation of $\pi_{1}(S)$, with limit set $\Lambda_{0} \cong S^{1}$. Then there is a continuous map $\phi: S^{1} \rightarrow \Lambda_{\Gamma}$, which takes the action by $\rho_{0}$ on $S^{1}$ to the action of $\rho$ on $\Lambda_{\Gamma}$.

In particular when the limit set is all of $\hat{\mathbf{C}}$, we obtain a sphere-filling curve which is invariant by the group action.

Finally we mention an immediate corollary of Theorem B:

Corollary C. If $\Gamma$ is a surface group satisfying $\mathrm{C} 2$, then $\Lambda_{\Gamma}$ is locally connected.

Plan of the paper. In order to go from the end invariants of a manifold to its isometry type, we first build a "model metric" which is quasi-isometric to the hyperbolic one, and which depends only on its end invariants. It then follows that two manifolds with the same end invariants are quasi-isometric to the same model, and Sullivan's rigidity theorem can be applied to show they are isometric.

The main difficulty is in controlling the simply degenerate ends. Such an end can be "filled up" by images of hyperbolic surfaces known as pleated surfaces, in such a way that control of the geometry of these surfaces is sufficient to control the geometry of the end. Our task is to obtain this control, starting only from the ending laminations.

The bulk of the proof of Theorem $A$ is in sections 4 and 5. Section 4 gives a careful discussion of families of homotopic pleated surfaces in hyperbolic 3manifolds, and shows in particular how their internal geometry controls their placement in the 3-manifold, and the homotopies between them. Section 5 utilizes this information, together with the main result of [Min93] (which is summarized in section 9), to construct a model manifold and the accompanying quasi-isometry to the hyperbolic manifold. Section 6 combines these results to give Theorem $\mathrm{A}$.

In section 7 we prove the above-mentioned extensions of the theorems of Cannon and Thurston, together with Theorem E. The main tool here is the model manifold constructed in section 5 , together with some basic facts about quasi-isometries in hyperbolic space. Section 8 contains the application of these to the proof of Theorem $\mathrm{E}$. 
Sections 2 and 3 are mostly expository. Section 2 gives some basic definitions and notation for Kleinian groups, surface theory, and Teichmüller theory. Section 3 is a discussion of quasi-isometries and related notions, and contains one useful but technical lemma about lifts of quasi-isometries to universal covers.

Section 9 is again mostly expository, giving a fuller discussion of basic facts about ending laminations (with proofs that are hard to find in the literature), as well as a summary of the results of [Min93] about families of pleated surfaces which are a key element of the proof in section 5 .

\section{NOTATION AND BACKGROUND}

2.1. Kleinian groups. A Kleinian group is a discrete subgroup $\Gamma$ of $\operatorname{Isom}\left(\mathbf{H}^{3}\right)$, which acts also by Möbius transformations on the Riemann sphere $\hat{\mathbf{C}}=\mathbf{C} U$ $\{\infty\}$. We assume throughout that $\Gamma$ is finitely generated, orientation preserving, and contains no elliptic (torsion) elements. Thus $N=\mathbf{H}^{3} / \Gamma$ is a complete orientable hyperbolic three-manifold, and $\Gamma \cong \pi_{1}(N)$.

The limit set of $\Gamma$, denoted $\Lambda=\Lambda_{\Gamma} \subset \hat{\mathbf{C}}$, is the smallest closed $\Gamma$-invariant subset of $\hat{\mathbf{C}}$. Let $\Omega=\Omega_{\Gamma}$ denote $\hat{\mathbf{C}}-\Lambda$, the domain of discontinuity. $\Gamma$ acts properly discontinuously on $\Omega$, and the quotient $\Omega / \Gamma$ is a Riemann surface, thought of as a "boundary at infinity" for $N$.

If $N$ is homeomorphic to the interior of a compact manifold (which is the case for the manifolds discussed here, and conjectured to be true for all quotients of finitely generated Kleinian groups), we say that $N$ is topologically tame. In any case, due to a general theorem of Scott (see [Sco73]), $N$ contains a compact core $K(N)$, that is, a compact codimension-0 submanifold whose inclusion in $N$ is a homotopy equivalence. Each component of $N-K$ is a neighborhood for a unique end (see Bonahon [Bon86]), which gives a one-to-one correspondence between the ends and the components of $\partial K$.

2.1.1. Geometrically finite ends and the domain of discontinuity. Restrict now to the case where $N$ has no cusps, or equivalently that $\Gamma$ has no parabolic elements, and assume $N$ satisfies C1-that is, $\pi_{1}(N)$ has no non-trivial free decomposition. This can be interpreted topologically: it is equivalent to the condition that the inclusion map $i_{*}: \pi_{1}(S) \rightarrow \pi_{1}(N)$ is injective for each component $S$ of $\partial K(N)$, or that $\partial K(N)$ is incompressible (see [Bon86]).

Let $C(N)$ denote the convex core of $N$, the smallest convex submanifold of $N$ whose inclusion is a homotopy equivalence. Alternately $C(N)$ is obtained as the quotient by $\Gamma$ of the convex hull $\mathscr{C} \mathscr{H}(\Lambda) \subset \mathbf{H}^{3}$ of the limit set. An end of $N$ is called geometrically finite if it has a neighborhood disjoint from $C(N) . N$ is called geometrically finite if every end of $N$ is geometrically finite (or equivalently, for the case without parabolics, if $C(N)$ is compact).

Let $e$ be a geometrically finite end of $N$, and $S_{e}$ the corresponding boundary of the convex core $C(N)$. Assuming that $S_{e}$ is incompressible, it lifts to a union of convex disks in the universal cover, each (intrinsically) isometric to a hyperbolic plane, and meeting $\hat{\mathbf{C}}$ in a Jordan curve. Let $\widetilde{S}_{e}$ denote one such 
disk, and $\Gamma_{e}$ its stabilizer in $\Gamma . \widetilde{S}_{e}$ bounds a (topological) halfspace on its convex side that meets $\hat{\mathbf{C}}$ in a disk component $\Omega_{e}$ of $\Omega$. The Poincaré metric on $\Omega_{e}$ descends to a hyperbolic metric $\rho_{e}$ on the quotient $\Omega_{e} / \Gamma_{e}$, which may be canonically identified with $S_{e}$, via orthogonal projection from $\Omega_{e}$ to $\widetilde{S}_{e}$ (see [EM87] and section 5).

The metric $\rho_{e}$ determines a point in the Teichmüller space $\mathscr{T}\left(S_{e}\right)$ (see section 2.2 for definitions). In the case of geometrically finite manifolds, this data, for all the boundary components of $K(N)$, serves to classify $N$ up to isometry (see [AB60, Ber70, Kra72, Mar74, Mas71]). We shall call the element $\left[\rho_{e}\right] \in \mathscr{T}\left(S_{e}\right)$ the end invariant of the geometrically finite end $e$.

2.1.2. Geometric tameness and ending laminations. Let $e$ be an end of $N$, with $S_{e}$ the corresponding component of $\partial K(N)$, and let $N_{e}$ be the neighborhood of $e$ bounded by $S_{e}$. If $e$ is not geometrically finite, we may still be able to control it via the following notion of Thurston (see [Thu82a, Bon86]).

Definition 2.1. An end $e$ of a manifold $N$ with no cusps that satisfies $\mathrm{Cl}$ is simply degenerate if there is a sequence $\left\{\gamma_{i}\right\}$ of simple closed curves on $S_{e}$, whose geodesic representatives $\gamma_{i}^{*}$ in $N$ are eventually contained in any neighborhood of $e$.

We say that the curves $\gamma_{i}^{*}$ "exit the end".

Remark. There is a slightly more complicated version of this definition which holds for manifolds with parabolics, and manifolds that do not satisfy $\mathrm{C1}$ (quotients of general purely hyperbolic free groups, for example). See [Can93] for a good discussion of these cases.

$N$ is called geometrically tame if all its ends are either geometrically finite or simply degenerate. Thurston showed that geometrically tame manifolds have some useful topological and analytical properties. In particular a geometrically tame manifold is topologically tame.

It is conjectured that all hyperbolic manifolds (with finitely generated fundamental group) are geometrically tame. The basic result in this direction which is relevant to us is the following:

Theorem 2.2 (Bonahon [Bon86]). If $N$ satisfies $\mathrm{C} 1$, then $N$ is geometrically tame.

Thurston defined an invariant of a simply degenerate end which characterizes its approximate geometry, and is an analogue of the conformal structure at infinity of a geometrically finite end. This invariant is called the ending lamination $\lambda_{e}$, which is an element of the geodesic lamination space $\mathscr{G} \mathscr{L}\left(S_{e}\right)$ (see section 2.2 for a definition of $\mathscr{L} \mathscr{L}\left(S_{e}\right)$ and the space $\mathscr{M} \mathscr{L}\left(S_{e}\right)$ of measured laminations). The ending lamination is defined by virtue of the following fact, which we state in the no-cusp case (see also [Bon, Can93] for a proof).

Lemma 2.3 (Thurston). Let $e$ be a simply degenerate end of a hyperbolic 3manifold without cusps, satisfying $\mathrm{C1}$. There is a unique $\lambda_{e} \in \mathscr{G} \mathscr{L}\left(S_{e}\right)$ such that 
any sequence of simple closed curves $\gamma_{i}$ in $S_{e}$ whose geodesic representatives exit the end converges in $\mathscr{M L}(S)$ to a lamination whose support is $\lambda_{e}$.

The ending lamination $\lambda_{e}$ has the property that it intersects transversely the support of every measured lamination on $S_{e}$ except for itself.

In general, by the "end invariant" of a geometrically tame end we mean its ending lamination if it is simply degenerate, or its conformal structure at infinity if it is geometrically finite. Thurston's ending lamination conjecture (Conjecture 1.1 ) is the statement that, for a geometrically tame hyperbolic manifold, the ending laminations and Riemann surfaces at infinity suffice to determine the manifold uniquely.

\subsection{Surface theory.}

2.2.1. Laminations and foliations. Let $S$ be a closed surface, and $\sigma$ a hyperbolic metric on $S$. A geodesic lamination on $(S, \sigma)$ is a closed disjoint union of complete simple geodesics in the metric $\sigma$. Let $\mathscr{G} \mathscr{L}(S)$ denote the space of all geodesic laminations on $(S, \sigma)$. Let $\mathscr{M L}(S)$ be the space of geodesic laminations on $(S, \sigma)$, equipped with transverse measures (see, e.g., [Thu82a, CB88, Cas82]). $\mathscr{M} \mathscr{L}(S)$ has a natural topology under which it is homeomorphic to a finite-dimensional cell. As the notation suggests, the choice of $\sigma$ is unimportant: a different choice would yield a canonically homeomorphic space (see [Hat88]). The simple closed geodesics, equipped with positive weights, form a dense subset of $\mathscr{M} \mathscr{L}(S)$. The length function $\ell_{\sigma}: \mathscr{L} \mathscr{L}(S) \rightarrow \mathbf{R}$ is continuous and linear under scaling of measures. The quotient $\mathscr{P} \mathscr{M} \mathscr{L}(S)=$ $(\mathscr{M L}(S)-\{0\}) / \mathbf{R}_{+}$, obtained by identifying laminations with the same support and proportional measures, is a sphere, and in particular compact.

We shall also be using an equivalent construction, that of measured foliations on a surface (see [FLP79]). Denote by $\mathscr{M F}(S)$ the space of equivalence classes of topological foliations on $S$ with three- or more-pronged saddle singularities, equipped with transverse measures. The equivalence is under isotopy, and collapsing of compact leaf segments joining singularities (Whitehead moves). There is a natural homeomorphism between $\mathscr{M F}(S)$ and $\mathscr{M L}(S)$, obtained by "straightening" each leaf of a foliation to its geodesic representative (see [Lev83] as well as a discussion in section 7). We form $\mathscr{P} \mathscr{M F}(S)$ by projectivizing, as above.

If $\mu$ is an element of $\mathscr{M L}(S)$ or $\mathscr{P} \mathscr{M L}(S)$, let the support $\operatorname{supp}(\mu)$ denote the element of $\mathscr{E} \mathscr{L}(S)$ obtained by forgetting the measure.

The geometric intersection number $i($,$) for closed curves on a surface ex-$ tends to a continuous function on $\mathscr{M L}(S) \times \mathscr{M} \mathscr{L}(S)$ (and thus on $\mathscr{M F}(S) \times$ $\mathscr{M F}(S)$ ), homogeneous in both arguments (see [Bon86]).

Two laminations $\lambda$ and $\mu$ in $\mathscr{E} \mathscr{L}(S)$ are said to fill up $S$ if the union of their geodesic representatives in any hyperbolic metric cuts $S$ into a union of disks each of whose boundaries is finitely many compact arcs of $\lambda$ and $\mu$ (see [Ker92, Thua]). We shall need the following facts about ending laminations in sections 5 and 7. 
Lemma 2.4. Let $\lambda$ be an ending lamination of an end $e$ of a hyperbolic 3manifold $N$ without cusps, satisfying $\mathrm{C} 1$. Then the following hold:

(1) Every half-leaf of $\lambda$ is dense in $\lambda$.

(2) If $\mu \neq \lambda$ is the support of a measured lamination then $\lambda$ and $\mu$ fill up $S_{e}$.

Proof. The support of any measured lamination decomposes into a disjoint union of minimal closed sublaminations (see [Lev83]). We claim that the ending lamination $\lambda$ is already minimal. For if not, let $\lambda_{1}$ and $\lambda_{2}$ be distinct minimal components of $\lambda$. A sufficiently small neighborhood of $\lambda_{1}$ must be disjoint from $\lambda_{2}$ and at least one of its boundary components $\gamma$ must be topologically non-trivial since $\lambda_{2}$ cannot be contained in a disk. Thus $\gamma$ has a geodesic representative disjoint from $\lambda$, in contradiction to the last assertion of Lemma 2.3.

Since any half-leaf of $\lambda$ must accumulate on a minimal component, we conclude that each half-leaf is dense.

For (2), let $U$ be a component of $S-(\lambda \cup \mu)$. If $U$ is not a topological disk then it contains a homotopically non-trivial curve, which again contradicts Lemma 2.3. Thus it is a disk, whose boundary consists of leaf segments of $\lambda$ and $\mu$. It remains to check that each segment is compact. A non-compact segment of $\lambda$ cannot occur because, being dense in $\lambda$, it would imply $\lambda \cap \mu=\varnothing$, contradicting Lemma 2.3. A non-compact segment of $\mu$ would accumulate on a minimal component of $\mu$ disjoint from $\lambda$, again contradicting Lemma 2.3.

2.2.2. Teichmüller space. The Teichmüller space $\mathscr{T}(S)$ of a closed surface $S$ is the space of conformal structures on $S$, considered up to conformal isomorphisms isotopic to the identity. Equivalently it is the space of hyperbolic metrics on $S$, up to isometries homotopic to the identity (see, e.g., [Abi80, Gar87]).

There is a natural topology on $\mathscr{T}(S)$, under which it is homeomorphic to a finite-dimensional cell (of the same dimension as $\mathscr{M L}(S)$ ). It has several natural metrics, and the one we shall be using throughout this paper is known as the Teichmüller metric. Given two points $\sigma, \tau \in \mathscr{T}(S)$, the distance between them is given as

$$
d_{T}(\sigma, \tau)=\frac{1}{2} \log K(\sigma, \tau),
$$

where $K$ is the least quasiconformal dilatation of a map from $(S, \sigma)$ to $(S, \tau)$ in the homotopy class of the identity.

The least dilatation is realized by a unique extremal map, known as a Teichmüller map, which has a special form: Begin with a holomorphic quadratic differential $\Phi$ on $(S, \sigma)$, that is, a tensor of the form $\Phi(z) d z^{2}$ in a holomorphic coordinate chart, where $\Phi(z)$ is holomorphic (see [Gar87, Str84]). Away from the zeros of $\Phi$ there is a canonical holomorphic coordinate $\zeta=x+i y$, defined locally up to translation and sign, such that $\Phi=d \zeta^{2}$ in this coordinate. The lines $\{y=c\}$ and $\{x=c\}$ are thus consistently defined, and form what are known as the horizontal and vertical foliations, respectively, or $\Phi_{h}$ and $\Phi_{v}$. The metric $|\Phi|=|d \zeta|^{2}=d x^{2}+d y^{2}$ is also canonically defined, and it is Eu- 
clidean with isolated singularities at the zeros of $\Phi$ where there is concentrated negative curvature. Note that the foliation leaves are geodesic with respect to $|\Phi|$. Let $\left|\Phi_{K}\right|$ denote the metric obtained from $|\Phi|$ by multiplying lengths of vertical leaves by $K^{-1 / 2}$ and horizontal leaves by $K^{1 / 2}$. This defines a new conformal structure $\tau$ and a quadratic differential $\Phi_{K}$ which is holomorphic in $\tau$, and the identity map is the unique extremal map.

Fixing the basepoint $\sigma$, the choice of all (unit-area) differentials $\Phi$ and dilatations $K$ parametrizes all of $\mathscr{T}(S)$. The set

$$
R(\sigma, \Phi)=\left\{\left[\left|\Phi_{K}\right|\right]: K \geq 1\right\}
$$

is a "Teichmüller geodesic ray". The foliation $\Phi_{v}$ is called the "endpoint" of the ray $R(\sigma, \Phi)$.

The following lemma, which follows from a compactness argument on the moduli space of surfaces and a theorem of Kerckhoff [Ker80], is proven in [Min93]:

Lemma 2.5. Let $\sigma$ and $\rho$ denote the hyperbolic metrics representing two points in $\mathscr{T}(S)$. Suppose that injectivity radii in $(S, \rho)$ and $(S, \sigma)$ are bounded below by $\epsilon>0$. Then

$$
d_{T}(\rho, \sigma)-b \leq \log \sup _{\gamma} \frac{\ell_{\sigma}(\gamma)}{\ell_{\rho}(\gamma)} \leq d_{T}(\rho, \sigma)+b,
$$

where $b$ depends on $\chi(S)$ and $\epsilon$. The supremum is taken over all homotopy classes of simple closed curves in $S$.

\section{QUASI-ISOMETRIES}

The notion of quasi-isometries in hyperbolic space and their extensions to the sphere at infinity is an important ingredient in the rigidity and deformation theory of hyperbolic manifolds, and has received much attention (and generalization) recently. In this section we shall briefly recall some of these ideas (see also [Can91, CDP90, GdlH90, Gro87]). Let

$$
P \sim_{(a, b)} Q
$$

(or simply $P \underset{\sim}{<}$ ) denote the inequality

$$
P \leq a Q+b
$$

with constants $a, b$ independent of the particular context (where necessary we will denote the dependencies explicitly). Similarly let

$$
P \simeq_{(a, b)} Q,
$$

or simply $P \simeq Q$, denote the conjunction of $P \sim_{(a, b)} Q$ and $Q \underset{\sim_{(a, b)}}{ } P$. 
Let $f: X \rightarrow Y$ be a map (not necessarily continuous) between two metric spaces. If

$$
d_{Y}\left(f(x), f\left(x^{\prime}\right)\right) \lesssim_{(K, \delta)} d_{X}\left(x, x^{\prime}\right)
$$

for $K, \delta>0$ and any $x, x^{\prime}$ in $X$, we say that $f$ is $(K, \delta)$-quasi-Lipschitz, or simply quasi-Lipschitz. If

$$
d_{X}\left(x, x^{\prime}\right) \simeq_{(K, \delta)} d_{Y}\left(f(x), f\left(x^{\prime}\right)\right)
$$

for $K, \delta>0$ and every $x, x^{\prime} \in X$ we say that $f$ is a $(K, \delta)$-quasi-isometry, or simply a quasi-isometry.

We call $f$ cobounded if $d_{Y}(y, f(X))$ is bounded for all $y \in Y$. If the bound is $L \geq 0$ we say $f$ is $L$-cobounded.

Clearly a composition of quasi-isometries is a quasi-isometry, and likewise for quasi-Lipschitz. It is also clear that, given a cobounded quasi-isometry $f: X \rightarrow Y$ there exists $g: Y \rightarrow X$, also a cobounded quasi-isometry, such that $d_{X}(x, g(f(x)))$ and $d_{Y}(y, f(g(y)))$ are bounded. We call $g$ a quasi-inverse of $f$.

Now consider a continuous map $f: X \rightarrow Y$ between path-metric spaces. We say that $f$ is a liftable $(K, \delta)$-quasi-isometry if any lift $\tilde{f}: \widetilde{X} \rightarrow \tilde{Y}$ to the universal covers is a $(K, \delta)$-quasi-isometry. We similarly define liftably quasi-Lipschitz.

It is easy to construct examples of continuous quasi-isometries which are not liftable. One soon sees that some further topological as well as geometric constraints must be imposed. We shall find the following lemma useful.

Lemma 3.1. Let $f: X \rightarrow Y$ be a continuous map between two locally compact, connected path-metric spaces, and let $\tilde{f}: \tilde{X} \rightarrow \tilde{Y}$ be a lift to the universal covers.

Given $K, L, \delta>0$ there exists $B_{0}=B_{0}(K, L, \delta)$ such that, if the following conditions hold, then $f$ is a liftable quasi-isometry, and $\tilde{f}$ is L-cobounded:

Q1. $f$ is an $L$-cobounded homotopy equivalence for some $L \geq 0$.

Q2. $f$ is a $(K, \delta)$-quasi-isometry.

Q3. $f$ is liftably $(K, \delta)$-quasi-Lipschitz.

Q4. There exist numbers $A>0$ and $B>B_{0}$ such that, given a deck translation $\alpha: \tilde{X} \rightarrow \tilde{X}$ and $\xi \in \tilde{X}$, if $d_{\tilde{Y}}(\tilde{f}(\xi), \tilde{f}(\alpha(\xi))) \leq B$ then $d_{\tilde{X}}(\xi, \alpha(\xi))$ $\leq A$.

Note that Q4 can be stated as a property of closed loops in $X$ and $Y$ : a sufficiently short loop in $Y$ through any point is homotopic, fixing that point, to the image of a short loop in $X$.

Proof. Let $p: \widetilde{X} \rightarrow X$ and $q: \tilde{Y} \rightarrow Y$ be the covering projections, so that $f \circ p=q \circ \tilde{f}$. Since $f$ is a homotopy equivalence, it is easy to see that if $y \in f(X)$ then all of $q^{-1}(y)$ is contained in $\tilde{f}(\tilde{X})$. Thus, since $f$ is $L$ cobounded it follows that $\tilde{f}$ is $L$-cobounded also. 
We shall choose $B_{0}=\left(K^{2}+1\right) 2 L+(K+1) \delta$. With this choice, the following is a consequence of $\mathrm{Q} 1-\mathrm{Q} 4$ :

Q5. There exist $B^{\prime}>2 L$ and $A^{\prime}>0$ such that, if $d_{\tilde{Y}}\left(\tilde{f}\left(\xi_{1}\right), \tilde{f}\left(\xi_{2}\right)\right) \leq B^{\prime}$ and $\xi_{1}, \xi_{2} \in \tilde{X}$, then $d_{\tilde{X}}\left(\xi_{1}, \xi_{2}\right) \leq A^{\prime}$.

In fact, choose any $B>B_{0}$, and let $B^{\prime}=(B-(K+1) \delta) /\left(K^{2}+1\right)$. Let $x_{i}=p\left(\xi_{i}\right), \eta_{i}=\tilde{f}\left(\xi_{i}\right)$, and $y_{i}=q\left(\eta_{i}\right)=f\left(x_{i}\right)$. Then $d_{Y}\left(y_{1}, y_{2}\right) \leq B^{\prime}$, so by Q2 we have $d_{X}\left(x_{1}, x_{2}\right) \leq K B^{\prime}+\delta$. Thus there is a lift $\xi_{2}^{\prime}$ of $x_{2}$ such that $d_{\tilde{X}}\left(\xi_{1}, \xi_{2}^{\prime}\right) \leq K B^{\prime}+\delta$. Let $\eta_{2}^{\prime}=\tilde{f}\left(\xi_{2}^{\prime}\right)$. Then, by Q3, $d_{\tilde{Y}}\left(\eta_{1}, \eta_{2}^{\prime}\right) \leq K^{2} B^{\prime}+$ $(K+1) \delta$, so that $d_{\widetilde{Y}}\left(\eta_{2}, \eta_{2}^{\prime}\right) \leq\left(K^{2}+1\right) B^{\prime}+(K+1) \delta=B$. Since $\xi_{2}$ and $\xi_{2}^{\prime}$ differ by a deck translation of $\tilde{X}$, it follows from Q4 that $d_{\widetilde{X}}\left(\xi_{2}, \xi_{2}^{\prime}\right) \leq A$. Thus $d_{\tilde{X}}\left(\xi_{1}, \xi_{2}\right) \leq A+K B^{\prime}+\delta$, proving Q5.

Now take any $\xi_{i} \in \tilde{X}, \eta_{i}=\tilde{f}\left(\xi_{i}\right)$. The inequality

$$
d_{\widetilde{Y}}\left(\eta_{1}, \eta_{2}\right) \underset{\sim(K, \delta)}{<} d_{\widetilde{X}}\left(\xi_{1}, \xi_{2}\right)
$$

is already provided by condition Q2. It remains to prove the opposite inequality.

Let $B^{\prime \prime}=B^{\prime}-2 L$, which is positive by the choice of $B^{\prime}$. By connecting $\eta_{1}$ to $\eta_{2}$ with a path of length $d_{\widetilde{Y}}\left(\eta_{1}, \eta_{2}\right)$ and suitably subdividing it, we may obtain a sequence $\eta_{1}=s_{0}, s_{1}, \ldots, s_{n}=\eta_{2}$, such that $d_{\widetilde{Y}}\left(s_{i}, s_{i+1}\right) \leq B^{\prime \prime}$ and $n \leq 1+d_{\widetilde{Y}}\left(\eta_{1}, \eta_{2}\right) / B^{\prime \prime}$. Since $\widetilde{f}$ is $L$-cobounded we may find $t_{i} \in \widetilde{f}(\tilde{X})$ such that $d\left(s_{i}, t_{i}\right) \leq L$, and thus $d\left(t_{i}, t_{i+1}\right) \leq 2 L+B^{\prime \prime}=B^{\prime}$. Now, by Q5, given preimages $u_{i} \in \tilde{f}^{-1}\left(t_{i}\right)$ we have $d_{\tilde{X}}\left(u_{i}, u_{i+1}\right) \leq A^{\prime}$. Thus (letting $u_{0}=\xi_{1}$ and $u_{n}=\xi_{2}$, and summing),

$$
d_{\tilde{X}}\left(\xi_{1}, \xi_{2}\right) \leq A^{\prime} n \leq \frac{A^{\prime}}{B^{\prime \prime}} d_{\widetilde{Y}}\left(\eta_{1}, \eta_{2}\right)+A^{\prime}
$$

Let us briefly describe an example that illustrates why the lower bound $B_{0}$ in condition Q4 is necessary. Let $G_{n}$ denote a graph on $n+1$ vertices labelled $c^{n}, a_{1}^{n}, \ldots, a_{n}^{n}$, with the following edges: $\left\{a_{i}^{n} a_{i+1}^{n}: i=1, \ldots, n-1\right\}$, which we color green, and $\left\{c^{n} a_{i}^{n}: i=1, \ldots, n\right\}$, which we color red (note that the edge $a_{1}^{n} a_{n}^{n}$ is not included). Put a metric on $G_{n}$ for which all edges have length 1. Now form the space $X$ from the union $\cup G_{n}$ by adjoining (red) edges $c^{n} c^{n+1}$ of length 1 .

Let $G_{n}(\epsilon)$ denote $G_{n}$ with the metric in which the red edges have length 1 and the green edges have length $\epsilon$. Form $Y$ from $\cup G_{n}(1 / n)$ by again adding (red) edges $c^{n} c^{n+1}$ of length 1 . Let $f: X \rightarrow Y$ be the identity map on the underlying graphs.

Properties Q1-Q3 certainly hold for $f$. In particular the fact that $f$ is a quasi-isometry follows from the observation that the diameters of $G_{n}$ (and $G_{n}(\epsilon)$ ) are always at most 3 . Condition Q4 would be satisfied, vacuously, if we 
could choose $B<2$, because every homotopically non-trivial loop in $Y$ must go through at least two red edges, and thus has length at least 2.

On the other hand, $\tilde{f}$ is not a quasi-isometry, because in $G_{n}$ there is always a non-trivial length-minimizing loop of length $n+1$ whose image has length $3-1 / n$ in $G_{n}(1 / n)$.

We will apply Lemma 3.1 in section 5 to a situation in which, in fact, a stronger condition than Q4 holds. Therefore we will only need the following weaker result:

Corollary 3.2. Let $f: X \rightarrow Y$ be a continuous map between two locally compact, connected path-metric spaces, and let $\widetilde{f}: \widetilde{X} \rightarrow \widetilde{Y}$ be a lift to the universal covers.

Suppose that conditions Q1-Q3 hold for $f$, as well as the following:

Q4'. For any $B>0$ there is an $A>0$ such that, given a deck translation $\alpha: \widetilde{X} \rightarrow \tilde{X}$ and $\xi \in \tilde{X}$, if $d_{\tilde{Y}}(\tilde{f}(\xi), \tilde{f}(\alpha(\xi))) \leq B$ then $d_{\tilde{X}}(\xi, \alpha(\xi)) \leq A$.

Then $\tilde{f}: \tilde{X} \rightarrow \tilde{Y}$ is a cobounded quasi-isometry.

We now consider quasi-isometries in the simpler context of surfaces.

Lemma 3.3. Let $\sigma$ be a hyperbolic metric on a closed surface $S$, with injectivity radii bounded below by $\epsilon>0$. Let $\Phi$ be a holomorphic quadratic differential of unit mass on $(S, \sigma)$. Then id : $(S, \sigma) \rightarrow(S,|\Phi|)$ is a liftable $(K, \delta)$-quasiisometry, where $K$ and $\delta$ depend only on $\epsilon$ and $\chi(S)$.

(Note that this is stronger than the fact that there is some map homotopic to the identity which is a liftable quasi-isometry.)

Proof. We first obtain a pointwise comparison between the hyperbolic metric $\sigma$ and the quadratic differential metric $|\Phi|$, using a standard type of compactness argument. Consider the set of triplets $\mathscr{Q}=\{(\mathscr{X}, p, \Phi)\}$, where $\mathscr{Z}$ is a Riemann surface of hyperbolic type, $p$ is a basepoint in $\mathscr{X}$, and $\Phi$ is a holomorphic quadratic differential on $\mathscr{Z}$. This space admits a geometric topology, described in [McM89, Appendix], which is an extension of the standard geometric topology on hyperbolic surfaces. In particular the ratio $|\Phi(p)| / \sigma(p)$ is well defined and continuous on $\mathscr{Q}$, where $\sigma$ is the (unique) hyperbolic metric on $\mathscr{Z}$. Let $\mathscr{Q}_{g}(\epsilon)$ denote the subset of $\mathscr{Q}$ for which $\mathscr{X}$ is a closed surface of genus $g$, and the injectivity radius of $\sigma$ is at least $\epsilon>0$ at every point. As a special case of Theorem A.3.1 of [McM89], the projectivized space $\mathbf{P} \mathscr{Q}_{g}(\epsilon)$ is compact, where $\mathbf{P} \mathscr{Q}_{g}(\epsilon)=\mathscr{Q}_{g}(\epsilon) / \mathbf{C}_{*}$, with $\mathbf{C}_{*}$ acting by multiplication on $\Phi$. Since $\mathbf{P} \mathscr{Q}_{g}(\epsilon)$ may be identified with the subset of $\mathscr{Q}_{g}(\epsilon)$ where the mass $\|\Phi\|$ is 1 , we may conclude that the function $|\Phi| / \sigma$ has an upper bound on this subset.

In our setting this implies that the map id : $(S, \sigma) \rightarrow(S,|\Phi|)$ is uniformly Lipschitz, and so is its lift to the universal cover.

The reciprocal ratio is not bounded, since $\Phi$ has zeros. However, the same compactness result implies that, fixing $\delta>0$, there is a uniform upper bound $\sigma(p) /|\Phi(p)| \leq K_{1}$ for $p$ lying outside a $\delta$-neighborhood $\mathscr{N}_{\delta}$ of the zeros of $\Phi$ 
(where the neighborhood is measured in the $\sigma$ metric). Thus the inverse of the identity is also uniformly Lipschitz outside $\mathscr{N}_{\delta}$.

The number of zeros of $\Phi$ is bounded by $2|\chi(S)|$, by the Poincare index theorem. It follows that the $\sigma$-diameter of a component of $\mathscr{N}_{3 \delta}$ is at most $L=12 \delta|\chi(S)|$. Therefore, given $\epsilon$, we may choose $\delta$ small enough so that each component of $\mathscr{N}_{3 \delta}$ is contained in a disk in $S$. Thus, any path $\alpha$ contained in $\mathscr{N}_{3 \delta}$ is homotopic (fixing endpoints) to a path of $\sigma$-length at most $L$.

Suppose $\ell_{|\Phi|}(\alpha) \leq 2 \delta / K_{1}$. If $\alpha$ lies outside $\mathscr{N}_{\delta}$ its $\sigma$-length is at most $2 \delta$. If not, then since the $|\Phi|$-distance from $\mathscr{N}_{\delta}$ to $S-\mathscr{N}_{3 \delta}$ must be at least $2 \delta / K_{1}$, it follows that $\alpha \subset \mathscr{N}_{3 \delta}$, so we may deform it to a bounded path, as above.

Since we can divide an arbitrary path into segments of $|\Phi|$-length at most $2 \delta / K_{1}$ and apply the above bounds, we conclude that id $:(S,|\Phi|) \rightarrow(S, \sigma)$ is liftably quasi-Lipschitz.

As an easy corollary of this we may obtain the following statement, which is well known:

Lemma 3.4. Let $\rho$ and $\sigma$ be two hyperbolic metrics on $S$, such that the injectivity radii $\operatorname{inj}_{\sigma}$ and $\operatorname{inj}_{\rho}$ are bounded below by $\epsilon>0$. Then there is a liftably quasi-isometric map $h:(S, \sigma) \rightarrow(S, \rho)$ isotopic to the identity, where the constants depend only on $\chi(S), d_{T}(\rho, \sigma)$, and $\epsilon$.

Proof. Let $\Phi$ be the quadratic differential on $(S, \sigma)$ associated to the Teichmüller map between $(S, \sigma)$ and $(S, \rho)$ isotopic to the identity. Let $\Phi_{K^{\prime}}$ be the image quadratic differential in $(S, \rho)$, where $K^{\prime}=\frac{1}{2} \log d_{T}(\rho, \sigma)$. Apply Lemma 3.3 to id : $(S, \sigma) \rightarrow(S,|\Phi|)$, and to id : $\left(S,\left|\Phi_{K}\right|\right) \rightarrow(S, \rho)$. Then compose these with the Teichmüller map, which is $K^{\prime}$-bilipschitz, to obtain the desired map.

3.1. Quasiconvexity and the sphere at infinity. The compactification of $\mathbf{H}^{3}$ by the Riemann sphere $\hat{\mathbf{C}}$ can be viewed as an intrinsic hyperbolic construction. One can in general compactify $\mathbf{H}^{n}$ using a "sphere at infinity" $S_{\infty}^{n-1}$ or $\partial \mathbf{H}^{n}$, by fixing a basepoint $x_{0}$ of $\mathbf{H}^{n}$, associating each $x \in \mathbf{H}^{n}$ to the geodesic segment from $x_{0}$ to $x$, and appending the sphere of infinite geodesic rays based at $x_{0}$, with the natural topology on the set of geodesics (this construction is independent of $x_{0}$ ). Isometries of $\mathbf{H}^{n}$ then act continuously on $\mathbf{H}^{n} \cup \partial \mathbf{H}^{n}$. (See [GdlH90] for this construction in the general context of "negatively curved" spaces in the sense of Cannon and Gromov.)

A subset $C$ of a path-metric space $X$ is $K$-quasiconvex if, given $x, y \in C$, any geodesic segment $\alpha$ whose endpoints are $x$ and $y$ is contained in a $K$ neighborhood of $C$. If $C$ is 0 -quasiconvex we say it is totally geodesic. The following is well known:

Theorem 3.5. If $C \subset X$ is $K$-quasiconvex and $h: X \rightarrow \mathbf{H}^{n}$ is a $(K, \delta)$-quasiisometry, then $h(C)$ is $K^{\prime}$-quasiconvex, where $K^{\prime}$ depends on $K, \delta$, and $n$.

An important special case (which in fact implies the general case) is when $C=X=\mathbf{R}$. In this case we say that $h(\mathbf{R})$ is a quasigeodesic, and the theorem 
implies that $h(\mathbf{R})$ lies in a uniform neighborhood of a true geodesic. Note also that it follows that $h(\mathbf{R})$ accumulates on the endpoints of this geodesic in $\partial \mathbf{H}^{n}$. Let us consider this phenomenon more generally.

Let $C \subset \mathbf{H}^{n}$ be any quasiconvex set, and let $\partial_{\infty} C$ denote the intersection with $\partial \mathbf{H}^{n}$ of the closure of $C$ in the compactification. If $F: C \rightarrow \mathbf{H}^{m}$ is any map, we say that $F$ extends to a map at infinity if there is a map $\partial F: \partial_{\infty} C \rightarrow$ $\partial \mathbf{H}^{m}$ such that, whenever $\lim x_{i}=x$ for $\left\{x_{i}\right\} \subset C$ and $x \in \partial_{\infty} C$,

$$
\lim _{i \rightarrow \infty} F\left(x_{i}\right)=\partial F(x) .
$$

The following theorem (see [GdlH90]) gives a sufficient (but not necessary) condition for this to occur:

Theorem 3.6. Let $C \subset \mathbf{H}^{n}$ be quasiconvex. A quasi-isometry $F: C \rightarrow \mathbf{H}^{m}$ extends to a map $\partial F$ at infinity, and $\partial F$ is continuous and injective.

In fact more is true. A proof of the following can also be found in [GdlH90].

Theorem 3.7. A quasi-isometry $F: \mathbf{H}^{3} \rightarrow \mathbf{H}^{3}$ extends to a quasiconformal homeomorphism $\partial F: \hat{\mathbf{C}} \rightarrow \hat{\mathbf{C}}$.

\section{HoMOTOPIES OF PLEATED SURFACES}

A pleated surface $(g, \rho)$ is a map $g:(S, \rho) \rightarrow N$, where $S$ is a surface equipped with complete hyperbolic metric $\rho, N$ is a hyperbolic manifold, and $g$ satisfies

(1) any $\rho$-rectifiable path in $S$ is taken by $g$ to a path in $N$ of equal length ( $g$ is a pathwise-isometry),

(2) there is a geodesic lamination $\lambda$ in $(S, \rho)$ such that $g$ maps leaves of $\lambda$ to geodesics, and is totally geodesic in $S-\lambda$.

See [Thua, CEG87] for more details. The smallest $\lambda$ which works in the definition is called the pleating locus of $g$. We think of the surface as being "bent" or "pleated" along this lamination, though this bending is complicated since the local leaf space of $\lambda$ is typically a Cantor set.

We sometimes refer to $(g, \rho)$ as just $g$, leaving $\rho$ implicit, noting that $\rho$ is in fact determined by $g$. We call $\rho$ the metric induced by $g$. Call $g$ incompressible if $g_{*}: \pi_{1}(S) \rightarrow \pi_{1}(N)$ is injective.

In this section we shall describe the information one can get about a family of homotopic pleated surfaces using simple arguments about homotopies and geodesics in hyperbolic manifolds. In particular we investigate the connection between the physical separation of the images of two pleated surfaces in a hyperbolic 3-manifold, and the distance between their induced hyperbolic metrics, as points in a Teichmüller space. It will also be important to consider how the lengths of the homotopies themselves are controlled by these distances, in order to understand the quasi-isometry that we will construct in the next section. Consider first the following definitions. 
Let $g: S \rightarrow N$ and $h: S \rightarrow N$ be two maps of a surface into a hyperbolic 3-manifold $N$. Define

$$
d_{N}(g, h)=\min \left\{d_{N}(x, y): x \in g(S), y \in h(S)\right\}
$$

where $d_{N}(x, y)$ denotes the distance between points in $N$.

Now let $H: S \times[0,1] \rightarrow N$ be a homotopy from $g$ to $h$, so that $H(\cdot, 0)=$ $g$ and $H(\cdot, 1)=h$. The length $\ell(H)$ of the homotopy is defined as the maximal length in $N$ of a trajectory $H_{p}=\{H(p, t): t \in[0,1]\}$ over all $p$ in $S$. Define

$$
\mathbf{d}_{N}(g, h)=\min _{H} \ell(H)
$$

where the minimum is taken over all homotopies between $g$ and $h$. Note that we may as well assume that $H$ is geodesic, in the sense that the trajectories $H_{p}$ are all geodesics, and that each trajectory is parametrized at constant speed. With this normalization, $H$ is unique given the maps $g$ and $h$ :

Lemma 4.1. Given homotopic incompressible maps $g$ and $h$ from a surface $S$ of genus at least 2 to a hyperbolic 3-manifold $N$, there is a unique homotopy class of homotopies between them. In particular, there is a unique (normalized) geodesic homotopy.

Proof. Suppose $H_{i}: S \times[0,1] \rightarrow N, i=1,2$, are two homotopies. Let $H_{2}^{-1}$ denote $\mathrm{H}_{2}$ traversed in the opposite direction, and let $T=H_{1} \circ H_{2}^{-1}$ denote the composition, which gives rise to a map $T: S \times S^{1} \rightarrow N$.

Fix a point $p \in S$, and let $T_{p}$ denote $T$ restricted to $\{p\} \times S^{1}$. We shall first prove that $T_{p}$ is null-homotopic. Let $\beta_{1}$ and $\beta_{2}$ be two non-commuting loops in $S$ based at $p$. The map $\left.T\right|_{\beta_{i} \times S^{1}}$ is a map of the torus into $N$. Since a hyperbolic manifold has no incompressible non-peripheral tori, $T_{*}\left(\pi_{1}\left(\beta_{i} \times S^{1}\right)\right)$ must lie in a maximal cyclic subgroup or a $\mathbf{Z} \oplus \mathbf{Z}$ subgroup corresponding to a cusp. Two such subgroups, if they are not equal, must have trivial intersection. Therefore if $T_{p}$ is non-trivial then $g\left(\beta_{1}\right)$ and $g\left(\beta_{2}\right)$ commute in $\pi_{1}(N)$, a contradiction to the choice of the $\beta_{i}$ and the assumption that $g$ is $\pi_{1}$-injective.

Thus each trajectory $T_{p}$ is null-homotopic. If $D$ is a disk and $S^{1}=\partial D$, it follows that $T$ extends continuously to $\hat{T}: S \times D \rightarrow N$ where $\left.\hat{T}\right|_{S \times S^{1}}=T$; we can define $\hat{T}$ on $\Sigma \times D$ for a 1-skeleton $\Sigma$ of $S$ because $\pi_{2}(N)=0$, and on all of $S \times D$ because $\pi_{3}(N)=0$. (Since $N$ is covered by a contractible space, $\pi_{k}(N)=0$ for all $k>1$.) The map $\hat{T}$ provides the desired homotopy between $H_{1}$ and $H_{2}$.

When the metrics $\rho$ and $\sigma$ of two homotopic pleated surfaces are similar, we expect the pleated surfaces to have a short homotopy. More precisely,

Lemma 4.2. Let $g:(S, \rho) \rightarrow N$ and $h:(S, \sigma) \rightarrow N$ be homotopic incompressible pleated surfaces, and suppose $\operatorname{inj}_{N}(x) \geq \epsilon>0$ for all $x \in N$. Given $K, \delta>0$ there exists $B=B(K, \delta, \chi(S), \epsilon)$ such that, if $\phi:(S, \rho) \rightarrow(S, \sigma)$ 
is a liftably $(K, \delta)$-quasi-Lipschitz map homotopic to the identity, then

$$
\mathbf{d}_{N}(g, h \circ \phi) \leq B
$$

Proof. After lifting, if necessary, to an appropriate cover of $N$, we may assume that $g$ and $h$ are isomorphisms on $\pi_{1}$ (note that $d_{N}$ is unchanged by a covering projection).

The injectivity radius condition on $N$ and the $\pi_{1}$-injectivity of $g$ implies that $\operatorname{inj}_{\rho}(x)>\epsilon$ for all $x \in S$. Thus, by standard methods of two-dimensional hyperbolic geometry, there is a bound $A(\chi(S), \epsilon)$ on $\operatorname{diam}_{\rho}(S)$, and there is a triangulation $T$ of $S$ with one vertex $v$, such that $\ell_{\rho}(\beta) \leq A$ for every edge $\beta$ of $T$.

Let $H: S \times[0,1] \rightarrow N$ be the geodesic homotopy between $g$ and $h \circ \phi$. We shall obtain a bound on the length of the trajectory $H_{v}$.

Fixing a loop $\beta$ in $T$ (that is, an edge meeting $v$ at both ends), let $\beta^{*}$ denote the geodesic representative in $N$ of $g(\beta)$. Consider the shortest homotopy between the map $g: \beta \rightarrow N$ and any parametrization of $\beta^{*}$. We can bound the length of this homotopy with the following well-known fact, which we shall use again a few times in this paper. For a homotopy $H$ let $\ell^{\prime}(H)$ denote the length of its shortest trajectory.

Lemma 4.3. Let $N$ be a hyperbolic manifold, $\alpha: S^{1} \rightarrow N$ a homotopically nontrivial closed curve, and $\alpha^{*}$ its geodesic representative. Let $F_{0}$ be the shortest among all homotopies $F: S^{1} \times[0,1] \rightarrow N$ such that $F(\cdot, 0)=\alpha(\cdot)$ and $F(\cdot, 1)$ is any parametrization of $\alpha^{*}$. The shortest trajectory length of $F_{0}$ is bounded by:

$$
\cosh \ell^{\prime}\left(F_{0}\right) \leq \ell(\alpha) / \ell\left(\alpha^{*}\right) .
$$

Proof (sketch). One can see this by considering the universal cover $\mathbf{H}^{3}$, where a lift $\widetilde{\alpha}^{*}$ of $\alpha^{*}$ is a geodesic, and the corresponding lift of $\widetilde{\alpha}: \mathbf{R} \rightarrow \mathbf{H}^{3}$ has image outside the $\ell^{\prime}\left(F_{0}\right)$-neighborhood of $\alpha^{*}$. The inequality follows from the fact that the shortest homotopy is obtained from the closest-point projection to $\tilde{\alpha}^{*}$, and this projection contracts by a factor of at least $\cosh R$ where $R$ is the distance to the geodesic.

Note also that $\ell\left(F_{0}\right) \leq \ell^{\prime}\left(F_{0}\right)+\frac{1}{2} \ell(\alpha)$, so that we obtain a bound on the length of the longest trajectory as well.

In our case, the injectivity radius condition implies that $\ell\left(\beta^{*}\right) \geq 2 \epsilon$, whereas $\ell(g(\beta))=\ell_{\rho}(\beta)$ is at most $A$. This gives us a homotopy $F_{0}$ from $g(\beta)$ to $\beta^{*}$, with a uniform bound on $\ell\left(F_{0}\right)$, depending only on $\chi(S)$ and $\epsilon$.

Since $\phi$ is assumed to be liftably $(K, \delta)$-quasi-Lipschitz, $\phi(\beta)$ is homotopic (rel $\phi(v)$ ) to a path $\beta^{\prime}$ such that $\ell_{\sigma}\left(\beta^{\prime}\right) \leq K A+\delta$. In fact, the homotopy can be chosen so that its trajectories have length bounded by $K A / 2+\delta$. This is because, lifting $\phi$ and $\beta$ to $\tilde{\phi}$ and $\tilde{\beta}$ in $\mathbf{H}^{2}$, we have for each $x \in \tilde{\beta}$ that $d(\widetilde{\phi}(x), \widetilde{\phi}(\partial \widetilde{\beta})) \leq K d(x, \partial \widetilde{\beta})+\delta \leq K A / 2+\delta$. We may apply Lemma 4.3 again to obtain a second uniformly bounded homotopy $F_{1}$ from $h\left(\beta^{\prime}\right)$ to some 
parametrization of $\beta^{*}$. The two parametrizations of $\beta^{*}$ may be different, but because $\ell\left(\beta^{*}\right) \leq A$ we can connect them by some short homotopy whose image lies in $\beta^{*}$.

Concatenating all of these homotopies, we obtain a homotopy $G: \beta \times$ $[0,1] \rightarrow N$ such that $G(\cdot, 0)=\left.g\right|_{\beta}, G(\cdot, 1)=\left.h \circ \phi\right|_{\beta}$, and $G\left(\cdot, \frac{1}{2}\right)$ parametrizes the geodesic $\beta^{*}$. We have a bound $\ell(G) \leq A^{\prime}(\chi(S), \epsilon)$ on the trajectory lengths of $G$.

Note, however, that $G$ may not be homotopic to the restriction of the global homotopy $H$ to $\beta \times[0,1]$ (see Figure 1). To detect how it may differ, we can make an argument similar to that in Lemma 4.1: The composition of $\left.H\right|_{\beta \times[0,1]}$ with $G^{-1}$ produces a map of the torus into $N$, which on the level of $\pi_{1}$ must have image in an abelian subgroup of $\pi_{1}(N)$, which must therefore be the cyclic subgroup generated by some primitive loxodromic element. Since $g_{*}$ is assumed to be an isomorphism on $\pi_{1}$ and $\beta$ is already primitive in $\pi_{1}(S)$, we conclude that $[g(\beta)]$ is this primitive element. Thus the trajectories $H_{v}$ and $G_{v}$ can only differ (up to homotopy rel endpoints) by a power of $[g(\beta)]$. We can use this fact to bound the length of $H_{v}$.

Given a lift $\widetilde{H}_{v}$ of $H_{v}$ to $\mathbf{H}^{3}$, let $\widetilde{G}_{v}$ be a lift of $G_{v}$ that shares an endpoint with $\widetilde{H}_{v}$. If $\widetilde{\beta}^{*}$ is the lift of $\beta^{*}$ that meets $\widetilde{G}_{v}$ then $\widetilde{H}_{v}$ is a geodesic segment lying in an $A_{2}$-neighborhood of $\widetilde{\beta}^{*}$, where $A_{2}=A_{2}(\chi(S), \epsilon)$ is the bound on the length of $G_{v}$. This is because $\widetilde{H}_{v}$ can be obtained from $\widetilde{G}_{v}$ by moving one of the endpoints by a translation along $\widetilde{\beta}^{*}$.

Some hyperbolic trigonometry tells us that, for a fixed $d_{0}$ and $t>0$, a $t$-neighborhood of $\widetilde{\beta}^{*}$ contains all of $\widetilde{H}_{v}$ except possibly for initial and final segments of length at most $L(t)=A_{2}+d_{0}+\log 1 / t$ (assuming $\ell\left(H_{v}\right)>2 L(t)$ ).

Now suppose for some $t>0, V>0$ that $\ell\left(H_{v}\right)>2 L(t)+V$. Applying the above paragraph to $\beta=\beta_{1}, \beta_{2}$ where $\beta_{1}$ and $\beta_{2}$ are distinct loops of $T$, we find a segment of $\widetilde{H}_{v}$ of length $A$ that lies within distance $t$ of two lifts, $\widetilde{\beta}_{1}^{*}$ and $\widetilde{\beta}_{2}^{*}$. It follows that a segment of $\widetilde{\beta}_{2}^{*}$ of length $V-2 t$ lies in a $2 t$-neighborhood of $\tilde{\beta}_{1}^{*}$.

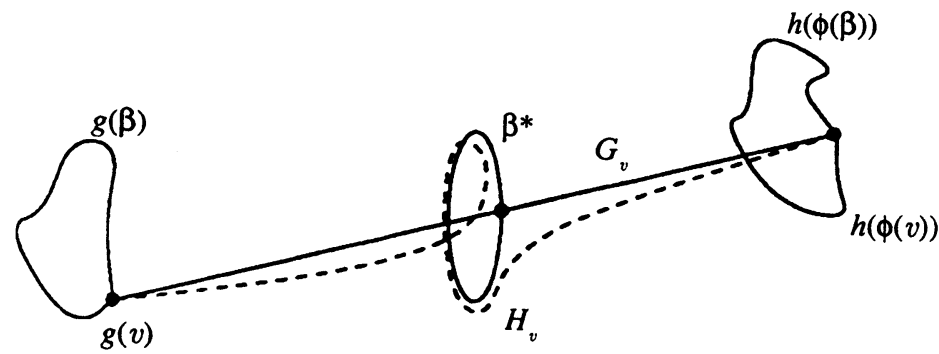

FIGURE 1. The short homotopy $G$ between two short loops $g(\beta)$ and $h(\phi(\beta))$ may differ from the global homotopy $H$, but only by a bounded power of $\beta$. 
Let $T_{1}$ and $T_{2}$ be the primitive elements in $\pi_{1}(N)$ whose axes are $\widetilde{\beta}_{1}^{*}$ and $\widetilde{\beta}_{2}^{*}$, and whose translation distances are $\ell_{1}, \ell_{2} \leq A$. There exist positive integers $m, n$ with $m \leq 2 \ell_{1} / \epsilon$ such that $\left|m \ell_{2}-n \ell_{1}\right| \leq \epsilon / 2$, where $\epsilon$ is the lower bound on injectivity radii in $N$ (just consider the distribution of the set $\left\{m \ell_{2}\left(\bmod \ell_{1}\right)\right\}$ in $\left.\left[0, \ell_{1}\right]\right)$.

It is not hard to see that, for sufficiently small $t$ and large $V$ (for instance $t<\epsilon / 12$ and $V>\epsilon / 6+2 A^{2} / \epsilon$ will do), it follows that the translation distance of one of $T_{1}^{n} T_{2}^{m}$ or $T_{1}^{-n} T_{2}^{m}$ is less than $\epsilon$; the idea is that there is a point $x \in \widetilde{\beta}_{2}^{*}$ such that $T_{2}^{k}(x)$ is in the $2 t$-neighborhood of $\widetilde{\beta}_{1}^{*}$ for $0 \leq k \leq m$. Thus we obtain a contradiction since $T_{1}^{ \pm n} T_{2}^{m}$ cannot be the identity, by choice of $\beta_{1}$ and $\beta_{2}$. We conclude that, in fact, $\ell\left(H_{v}\right) \leq 2 L(t)+V$.

A bound on $\ell(H)$ is now easy to obtain. For any $p \in S$, let $\alpha$ be a path from $p$ to $v$ of length $\ell_{\rho}(\alpha) \leq A$. By hypothesis, $\phi(\alpha)$ is homotopic rel endpoints to $\alpha^{\prime}$ such that $\ell_{\sigma}\left(\alpha^{\prime}\right) \leq K A+\delta$. The trajectory $H_{p}$ is geodesic, and homotopic rel endpoints to the concatenation $g(\alpha) \cdot H_{v} \cdot h\left(\alpha^{\prime}\right)^{-1}$, so its length is bounded by $2 L(t)+V+(K+1) A+\delta$.

The following result is a slight strengthening of Lemma 4.5 in [Min93]:

Lemma 4.4. Fix $S$ and $\epsilon>0$. Given $B>0$ there exists $A$ such that if $g:(S, \rho) \rightarrow N$ is a pleated surface, $g_{*}$ is an isomorphism on $\pi_{1}$, and injectivity radii in $N$ are bounded below by $\epsilon$, then the following holds:

Let $\alpha \subset S$ be a closed curve through $x \in S$, p-geodesic except possibly at $x$, and let $\beta$ denote the shortest curve in $N$ passing through $g(x)$ and homotopic to $g(\alpha)$. Then

$$
\ell_{N}(\beta) \leq B \quad \Longrightarrow \quad \ell_{\rho}(\alpha) \leq A .
$$

Proof (sketch). This lemma differs from the one proven in [Min93] in two respects. First, the curve $\alpha$ is assumed simple in the original lemma, but this fact is not actually used in the proof (I am grateful to the referee for pointing this out). Second, $\alpha$ is taken to be the closed $\rho$-geodesic in a free homotopy class, rather than a homotopy class with basepoint. However this is also only a slight perturbation of the proof. The idea of the original proof is to use a compactness argument on the space of all pleated maps and hyperbolic manifolds of the specified type. A counterexample to the lemma would yield a convergent sequence of examples, and in the limit both $\beta$ and $\alpha$ necessarily have finite lengths. One can then use this fact to obtain a uniform bound for the sequence, which is a contradiction. It is easy to see that the same logic works when one also keeps track of the basepoints.

Thus, the geometry of a pleated surface serves as a coarse approximation to the geometry of the manifold nearby. A consequence of this is the following:

Lemma 4.5 (Corollary 4.6 of [Min93]). Given $a>0$ there exists $b(a, \chi(S), \epsilon)$ such that if $g:(S, \rho) \rightarrow N$ and $h:(S, \sigma) \rightarrow N$ are homotopic pleated surfaces 
which are isomorphisms on $\pi_{1}$ and $N$ satisfies $\mathrm{C} 2$, then

$$
d_{N}(g, h) \leq a \quad \Longrightarrow \quad d_{T}(\sigma, \rho) \leq b
$$

Given a diffeomorphism $\psi: S \rightarrow S$ isotopic to the identity, it is natural, especially in the context of Teichmüller space, to consider $\left(g \circ \psi, \psi^{*} \rho\right)$ to be equivalent to $(g, \rho)$, and we then write $g \sim g \circ \psi$, and similarly for $(h, \sigma)$. Thus the quantity of actual interest to us is

$$
\tilde{\mathbf{d}}_{N}(g, h)=\inf \left\{\mathbf{d}_{N}\left(g, h^{\prime}\right): h \sim h^{\prime}\right\}
$$

(note that it is unnecessary to vary both $g$ and $h$, since $\mathbf{d}_{N}(g \circ \psi, h \circ \psi)=$ $\left.\mathbf{d}_{N}(g, h)\right)$.

Now, given a bound on $d(g, h)$, we may apply Lemma 4.5 to bound the Teichmüller distance between the induced metrics $\rho$ and $\sigma$, then by Lemma 3.4 obtain a liftably quasi-Lipschitz map $\phi:(S, \rho) \rightarrow(S, \sigma)$, and finally apply Lemma 4.2 to bound $\mathbf{d}_{N}(g, h \circ \phi)$. Thus we have:

Corollary 4.6. Given $A>0$ there exists $B(A, \chi(S), \epsilon)$ such that if $g, h$ are homotopic pleated surfaces inducing isomorphisms on $\pi_{1}$ and $N$ satisfies C2, then

$$
d_{N}(g, h) \leq A \quad \Longrightarrow \quad \tilde{\mathbf{d}}_{N}(g, h) \leq B
$$

We extend this to a more global statement in the following two lemmas.

Lemma 4.7. If $N$ satisfies $\mathrm{C} 2$ and the pleated surfaces $g, h: S \rightarrow N$ induce isomorphisms on $\pi_{1}$, then

$$
\widetilde{\mathbf{d}}_{N}(g, h) \simeq_{(a, b)} d_{N}(g, h)
$$

where $a, b>0$ depend only on $\chi(S)$ and the injectivity radius bound $\epsilon$.

Proof. The inequality $d_{N} \leq \tilde{\mathbf{d}}_{N}$ is obvious.

Let $g, h$ be pleated surfaces as above, with $d_{N}(g, h)=R$. Thurston showed that one consequence of the geometric tameness of $N$ is that every point in the convex hull of $N$ is within a uniformly bounded distance $A$ of the image of a pleated surface homotopic to $g$ and $h$ (see [Thu82a, §9.5], and also a brief discussion in [Min93, Theorem 3.5]). Moreover, the image of any pleated surface always lies in the convex hull. Thus we can connect $g(S)$ to $h(S)$ with a geodesic of length $R$, subdivide it and obtain a sequence $g=g_{1}, g_{2}, \ldots, g_{n}=$ $h$ of pleated surfaces such that $d_{N}\left(g_{i}, g_{i+1}\right) \leq 3 A$ and $n \leq R / A+1$. It will therefore suffice to find a uniform bound on $\widetilde{\mathbf{d}}_{N}\left(g_{i}, g_{i+1}\right)$, since it is easy to see that $\tilde{\mathbf{d}}_{N}(g, h) \leq \sum \tilde{\mathbf{d}}_{N}\left(g_{i}, g_{i+1}\right)$.

Thus we have essentially reduced to the case that $d_{N}(g, h) \leq 3 A$, which follows from Corollary 4.6.

We can also extend Lemma 4.5 to large distances: 
Lemma 4.8. If $g:(S, \rho) \rightarrow N$ and $h:(S, \sigma) \rightarrow N$ are homotopic incompressible pleated surfaces then

$$
d_{T}(\rho, \sigma) \simeq_{(a, b)} \tilde{\mathbf{d}}_{N}(g, h)
$$

with constants depending only on $\chi(S), \epsilon$.

This lemma should be compared with Theorem 9.4, which was proved in [Min93]. That theorem implies that the set of metrics $\rho$ obtained from pleated surfaces homotopic to $g$ lies in a bounded neighborhood of some Teichmüller geodesic in $\mathscr{T}(S)$, whereas Lemma 4.8 can be interpreted as describing some kind of "efficiency" in the way the pleated surface metrics move through $\mathscr{T}(S)$ as the surfaces move through $N$. Neither result is contained in the other, however. We should note that Lemma 4.8 has been observed before by McMullen, although not written down.

Proof. Again, if necessary lift $g, h$, and the homotopy $H$ to a cover, so that we may assume that $g_{*}=h_{*}: \pi_{1}(S) \rightarrow \pi_{1}(N)$ is an isomorphism, and $N$ is homeomorphic to $S \times \mathbf{R}$.

Lemma 4.5 together with the obvious inequality $d_{N} \leq \tilde{\mathbf{d}}_{N}$ implies that given $A$ there is a $B$ such that

$$
\tilde{\mathbf{d}}_{N}(g, h) \leq A \quad \Longrightarrow \quad d_{T}(\rho, \sigma) \leq B
$$

Using a sequence of intermediate surfaces as in the previous lemma, we can therefore obtain

$$
d_{T}(\rho, \sigma) \leq \frac{B}{A} \widetilde{\mathbf{d}}_{N}(g, h)+B
$$

On the other hand, if $d_{T}(\rho, \sigma)=D$ then, by Lemma 2.5 ,

$$
\frac{\ell_{\sigma}(\gamma)}{\ell_{\rho}(\gamma)} \leq c e^{D}
$$

for $c=c(\chi(S), \epsilon)$ (where $\gamma$ is any essential curve in $S$ and $\ell$ here refers to length of geodesic representative). Fix $\gamma$ in $S$, and let $\gamma^{*}$ be the geodesic representative of $g(\gamma)$ in $N$. Then, for any curve $\gamma^{\prime}$ homotopic to $\gamma^{*}$ it follows, as in Lemma 4.3, that

$$
\ell_{N}\left(\gamma^{\prime}\right) \geq \ell_{N}\left(\gamma^{*}\right) \cosh r\left(\gamma^{\prime}\right)
$$

where $r\left(\gamma^{\prime}\right)=d_{N}\left(\gamma^{\prime}, \gamma^{*}\right)$. We can choose $\gamma$ so that $\ell_{N}(g(\gamma))=\ell_{\rho}(\gamma) \leq c_{1}$, for some $c_{1}(\chi(S))$, and it then follows, since $\ell_{N}\left(\gamma^{*}\right) \geq \epsilon$, that

$$
r(g(\gamma)) \leq c_{2}=\left|\cosh ^{-1}\left(c_{1} / \epsilon\right)\right|
$$


Let $\gamma^{\prime \prime}$ be the $\sigma$-geodesic homotopic to $\gamma$. Applying (4.2) with $\gamma^{\prime}=h\left(\gamma^{\prime \prime}\right)$ to the length-ratio bound of (4.1), we obtain

$$
\begin{aligned}
c e^{D} & \geq \frac{\ell_{N}\left(\gamma^{*}\right) \cosh r\left(h\left(\gamma^{\prime \prime}\right)\right)}{\ell_{\rho}(\gamma)} \\
& \geq \frac{\epsilon}{c_{1}} \cosh r\left(h\left(\gamma^{\prime \prime}\right)\right) .
\end{aligned}
$$

Thus

$$
\begin{aligned}
d_{N}(g, h) & \leq r\left(h\left(\gamma^{\prime \prime}\right)\right)+r(g(\gamma)) \\
& \leq D+\log \left(2 c c_{1} / \epsilon\right)+c_{2} .
\end{aligned}
$$

An application of Lemma 4.7 concludes the proof.

\section{THE MODEL MANIFOLD}

In this section we shall prove the main structure theorem that leads to Theorem A.

Theorem 5.1 (The model manifold). Let $N$ be a hyperbolic manifold satisfying C1-2, and let $e_{1}, \ldots, e_{k}$ be the ends of $N$. There exists a manifold $M$ homeomorphic to $N$, with a path-metric which depends only on the homeomorphism type of $N$ and on the end invariants $\left\{\lambda_{e_{i}}\right\}$, together with a map $f: M \rightarrow N$ homotopic to a homeomorphism, whose lift $\tilde{f}: \widetilde{M} \rightarrow \mathbf{H}^{3}$ is a cobounded (in fact surjective) quasi-isometry.

Proof. Let $K \subset N$ be a compact core. For each end $e$ of $N$, let $S_{e}$ be the corresponding component of $\partial K$, and for later convenience let us assume $K$ has been chosen so that, if $e$ is geometrically finite, then $S_{e}$ is the corresponding boundary component of the convex core $C(N)$. Let $M_{e}=S_{e} \times[-1, \infty)$. The manifold $M$ will be the union

$$
M=K \cup \bigcup_{e} M_{e}
$$

under the identification $S_{e}=S_{e} \times\{-1\}$, with a path metric $s$ that we shall specify.

We will give $s$ by specifying an infinitesimal (singular) metric $d s$ (actually $d s$ will only be singular along a finite number of singular lines, in a controlled way). On $K$, let $d s$ be any fixed Riemannian metric. Define $f$ on $K$ to be the inclusion map into $N$.

To define the metric (and $f$ ) on the ends $M_{e}$ we need to consider separately the cases of geometrically finite and simply degenerate ends.

Geometrically finite ends. If $e$ is a geometrically finite end of $N$, the geometry of a neighborhood of $e$ is adequately modeled, for our purposes, by the neighborhood of an end of a Fuchsian group. Let us describe this explicitly, using a construction described in detail in [EM87]. 
Recall that we have chosen $S_{e} \subset \partial K(N)$ to be convex, and let $N_{e}$ denote the component of $N-K$ bounded by $S_{e}$. Let $\widetilde{S}_{e}$ be a component of the lift of $S_{e}$ to $\mathbf{H}^{3}$, and $\Omega_{e}$ the corresponding component of $\Omega$, as in section 2. Let $\tilde{N}_{e}$ denote the half-space in $\mathbf{H}^{3}$ between $\tilde{S}_{e}$ and $\Omega_{e}$.

Let $\Pi: \tilde{N}_{e} \rightarrow \widetilde{S}_{e}$ denote the nearest-point retraction, which descends to a retraction $\pi: N_{e} \rightarrow S_{e}$. Furthermore, $\Pi$ extends to infinity, to give a homeomorphism $\Pi: \Omega_{e} \rightarrow \widetilde{S}_{e}$.

Let $\widetilde{T}_{e}(t)$ denote the equidistant surface at distance $t$ from $\widetilde{S}_{e}$ in $\widetilde{N}_{e}$, and let $T_{e}(t)$ be its image in $N_{e}$. The surfaces $\widetilde{T}_{e}$ foliate $\tilde{N}_{e}$, and $\Pi$ restricted to each one is a homeomorphism. Let $\Pi_{t}: \Omega_{e} \rightarrow \widetilde{T}_{e}(t)$ denote the extension to $\Omega_{e}$ of the closest-point projection to one of these level surfaces, and note that $\Pi \circ \Pi_{t}=\Pi$. The induced hyperbolic metric on $\widetilde{T}_{e}(t)$ is related to the Poincaré metric $\tilde{\rho}_{e}$ on $\Omega_{e}$ by the following result (see [EM87, Theorem 2.3.1]):

Theorem 5.2 (Epstein-Marden). The map $\Pi_{t}$ is (cotht)-quasiconformal, $(4 \cosh t)$-Lipschitz, and its inverse $\Pi_{t}^{-1}: \widetilde{T}_{e}(t) \rightarrow \Omega_{e}$ is $(1 / \sinh t)$-Lipschitz.

(The theorem assumes only that $\Omega_{e}$ is simply-connected, which follows in our case from condition C1. Note also that in [EM87] the Lipschitz condition on $\Pi_{t}^{-1}$ is stated for $t<\log 2$, but in fact it holds without restriction, as one can see directly from the proof.)

In other words, the metric on each level surface is close to a multiple of the hyperbolic metric $\tilde{\rho}_{e}$ by a factor exponential in $t$. This suggests that we make the following definition for the model metric in $S_{e} \times[0, \infty)$ :

$$
d s^{2}=d t^{2}+(\cosh t)^{2} d \rho_{e}^{2}
$$

where $t \in[0, \infty)$ is the coordinate in the second factor of $M_{e}$, and $\rho_{e}$ is the metric induced by $\tilde{\rho}_{e}$ on the quotient of $\Omega_{e}$ which is naturally identified with $S_{e}$.

Extend $d s$ to $S_{e} \times(-1,0)$ in any continuous way, using a standard partition of unity argument.

We then define the map $f$ as follows. Let $\pi_{t}:\left(S_{e}, \rho_{e}\right) \rightarrow T_{e}(t)$ denote the map induced by $\Pi_{t}$ on the quotients. Then for $(p, t) \in S_{e} \times[1, \infty)$ set

$$
f(p, t)=\pi_{t}(p) .
$$

Extend $f$ in any continuous way to $S_{e} \times(-1,1)$. Theorem 5.2 implies that $f$ in $S_{e} \times[1, \infty]$ is a bilipschitz homeomorphism to its image (with a constant of about 4). Since $S_{e} \times[-1,1]$ is compact, it is clear that

Lemma 5.3. $f$ is a liftable quasi-isometry on $M_{e}$, for a geometrically finite end $e$.

It will be useful to observe that the metric in (5.1) is in fact exactly isometric to an end of a Fuchsian group. In fact, let $\Gamma_{0} \cong \pi_{1}\left(S_{e}\right)$ be a Fuchsian group that 
uniformizes $\left(S_{e}, \rho_{e}\right)$. The convex hull of $\Lambda_{\Gamma_{0}}$ is a plane, and a geodesic halfspace $H$ bounded by this plane may be parametrized as above, with (5.1) giving the hyperbolic metric. Thus we may identify $H$ with $\widetilde{S}_{e} \times(0, \infty)$ and let $H_{1} \subset$ $H$ be the subset corresponding to $\widetilde{S}_{e} \times(1, \infty)$. Now the map $f: H_{1} / \Gamma_{0} \rightarrow N_{e}$ lifts to a map $\tilde{f}: H_{1} \rightarrow \tilde{N}_{e}$ which preserves the product structure induced by the projections in both halfspaces. In particular, $\widetilde{f}$ extends naturally to a map $\widetilde{f}_{\infty}: \Omega_{0}^{+} \rightarrow \Omega_{e}$, where $\Omega_{0}^{+}$is the component of $\Omega_{\Gamma_{0}}$ bounding $H$, and by construction $\tilde{f}_{\infty}$ is an isometry of the Poincare metrics on the two domains. We record this as

Lemma 5.4. The map $\left.f\right|_{S_{e} \times(0, \infty)}$, viewed as a map from an end of the Fuchsian manifold $\mathbf{H}^{3} / \Gamma_{0}$ and lifted to the universal cover, extends to a map $\tilde{f}_{\infty}: \Omega_{0}^{+} \rightarrow$ $\Omega_{e}$ which is an isometry of the Poincare metrics, and in particular conformal.

Geometrically infinite ends. The case where $e$ is geometrically infinite is the most interesting, and is where the tools we have developed need to be applied. Let $\lambda_{e} \in \mathscr{G} \mathscr{L}\left(S_{e}\right)$ be the ending lamination for $e$. We will see in the appendix (section 9) that if $N$ admits a positive lower bound on the injectivity radius then the geometry of the end $e$ is modeled by a Teichmüller ray whose ending foliation corresponds (after straightening leaves) to $\lambda_{e}$. In fact, $\lambda_{e}$ turns out to be uniquely ergodic - it supports a unique transverse measure up to scaling, and thus this Teichmüller ray is unique up to choice of basepoint. The precise statement we will need is the following:

Theorem 5.5 (Ending ray). Fix a compact 3-manifold $\bar{N}$ whose interior $N_{0}$ admits hyperbolic structures, and such that $\pi_{1}(\bar{N})$ is indecomposable (condition C1). For each boundary component $S_{e}$ of $\frac{1}{N}$ (where e denotes the corresponding end of $\left.N_{0}\right)$, fix a basepoint $\left[\sigma_{e}\right]$ in $\mathscr{T}\left(S_{e}\right)$.

If $N$ is a hyperbolic manifold homeomorphic to $N_{0}$ and satisfying $\mathrm{C} 2$, then each simply degenerate end $e$ of $N$ gives rise to a unique Teichmüller ray $R\left(\sigma_{e}, \Phi_{e}\right)$, where the vertical foliation of $\Phi_{e}$ is equivalent to the ending lamination $\lambda_{e}$, and the following holds: For every point $\left[\sigma_{e}(t)\right]$ on the ray $R$, there is a pleated surface $g_{t}:\left(S_{e}, \rho_{e}(t)\right) \rightarrow N$ homotopic to the inclusion of $S_{e}$, such that $\left[\rho_{e}(t)\right]$ is a point in $\mathscr{T}\left(S_{e}\right)$ whose Teichmüller distance from $\left[\sigma_{e}(t)\right]$ is bounded by a constant $A$, which depends only on $\chi\left(S_{e}\right)$ and the lower bound $\epsilon$ on injectivity radius in $N$.

This theorem is essentially the main result of [Min93], with the uniqueness of the ray being a consequence of work of Masur. The details will be given in section 9. We shall now use the family of structures $\left[\sigma_{e}(t)\right]$ to construct a model metric on $M_{e}$, and then piece together the pleated maps $g_{t}$ to make the map $f$.

Let us then fix a basepoint $\left[\sigma_{e}\right]$ for $\mathscr{T}\left(S_{e}\right)$, and let $R=R\left(\sigma_{e}, \Phi_{e}\right)=\left\{\sigma_{e}(t)\right\}$ be as in Theorem 5.5, where $t \in(0, \infty)$ denotes arclength in the Teichmüller metric. 
The quadratic differential $\Phi_{e}$ gives rise to a singular euclidean metric $\left|\Phi_{e}\right|$ on $S_{e}$, which we can write infinitesimally as

$$
\left|\Phi_{e}\right|^{2}=d x^{2}+d y^{2}
$$

away from the zeros of $\Phi_{e}, d x$ being the measure in the horizontal direction and $d y$ in the vertical direction of $\Phi_{e}$ (that is, $d x$ is the transverse measure for $\Phi_{e, v}$ ).

Normalizing so that the identity map from $\left(S_{e}, \sigma_{e}\right)$ to $\left(S_{e}, \sigma_{e}(t)\right)$ is the Teichmüller map, the image quadratic differential $\Phi_{e}(t)$ induces a metric $\left|\Phi_{e}(t)\right|$, given by

$$
\left|\Phi_{e}(t)\right|=e^{2 t} d x^{2}+e^{-2 t} d y^{2}
$$

away from the zeros of $\Phi_{e}$ (see Figure 2).

Thus, we define the metric $d s$ on $S_{e} \times[0, \infty)$ via

$$
d s^{2}(p, t)=\left|\Phi_{e}(t)(p)\right|+d t^{2}
$$

where $p \in S_{e}$ and $t$ is the coordinate in $[0, \infty)$. Note that the singularities of this metric are the set $\Sigma \times[0, \infty)$, where $\Sigma$ is the (finite) set of zeros of $\Phi_{e}$.

As before, extend $d s$ in any continuous way to the remaining $S_{e} \times[-1,0]$.

We can now define $f$ on $M_{e}$, using the results of section 4. For integer $n \geq 0$, let

$$
g_{n}:\left(S_{e}, \rho_{e}(n)\right) \rightarrow N
$$

be one of the pleated surfaces promised by Theorem 5.5 (Ending ray).

Since $d_{T}\left(\sigma_{e}(n), \rho_{e}(n)\right) \leq A$, Lemma 3.4 implies the existence of a map $h_{n}:\left(S_{e}, \sigma_{e}(n)\right) \rightarrow\left(S_{e}, \rho_{e}(n)\right)$ which is isotopic to the identity and liftably

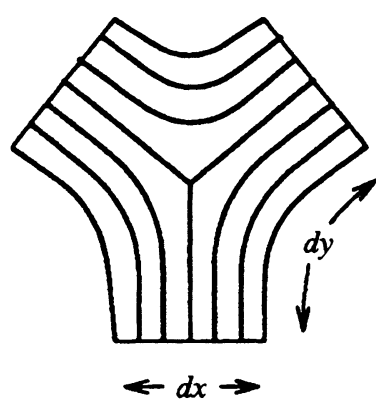

$t=0$

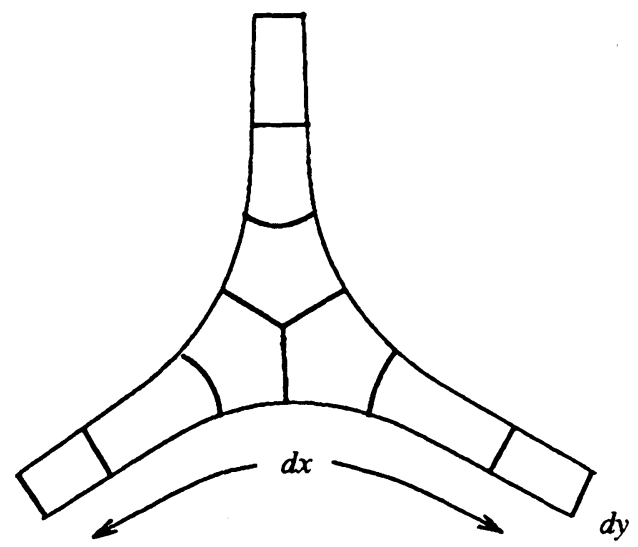

$t>0$

FIGURE 2. The metric $\left|\Phi_{e}(t)\right|$ for different values of $t$, near a singularity. The leaves shown are of the foliation $\Phi_{e, v}$. 
quasi-Lipschitz, with constants depending on $\chi(S), A$, and $\epsilon$. We may in fact assume that $h_{n}=\mathrm{id}$, for otherwise we can just replace $\rho_{e}(n)$ with $h_{n}^{*} \rho_{e}(n)$, and $g_{n}$ with $g_{n} \circ h_{n}$. Having normalized in this way, we set

$$
f(p, n)=g_{n}(p) .
$$

We may now deduce the following facts:

Lemma 5.6. The identity on $S_{e}$ is a liftable $(K, \delta)$-quasi-isometry, with respect to the following metrics, where $K$ and $\delta$ are independent of $n$ :

$$
\text { id }:\left(S, \rho_{e}(n)\right) \rightarrow\left(S, \rho_{e}(n+1)\right) \text {. }
$$

Similarly, the map

$$
\left.f\right|_{S_{e} \times\{n\}}:\left(S_{e},\left|\Phi_{e}(n)\right|\right) \rightarrow N
$$

is liftably quasi-Lipschitz, with constants independent of $n$.

Proof. First, the map

$$
\text { id : }\left(S_{e},\left|\Phi_{e}(n)\right|\right) \rightarrow\left(S_{e},\left|\Phi_{e}(n+1)\right|\right)
$$

is bilipschitz with constant $\exp (2)$, by the definition of $\left|\Phi_{e}(t)\right|$ in (5.2). Moreover, by Lemma 3.3,

$$
\mathrm{id}:\left(S_{e},\left|\Phi_{e}(t)\right|\right) \rightarrow\left(S_{e}, \sigma_{e}(t)\right)
$$

is a liftable $\left(K^{\prime}, \delta\right)$-quasi-isometry, for uniform $K^{\prime}$ and $\delta$. Since, by our normalization the identity is also uniformly liftably quasi-isometric from $\left(S_{e}, \sigma_{e}(k)\right)$ to $\left(S_{e}, \rho_{e}(k)\right)$ (for $k=n$ or $\left.n+1\right)$, the first claim follows.

The second claim follows from the same chain of facts, expressing $f$ as a composition of the identity from $\left(S_{e},\left|\Phi_{e}(n)\right|\right)$ to $\left(S_{e}, \sigma_{e}(n)\right)$ to $\left(S_{e}, \rho_{e}(n)\right)$, and finally using the fact that the pleated surface $g_{n}:\left(S_{e}, \rho_{e}(n)\right) \rightarrow N$ is 1-Lipschitz.

Lemma 4.2, together with the first claim of Lemma 5.6, implies that for each $n \geq 0$ there is a (unique) geodesic homotopy $H_{n}: S_{e} \times[0,1] \rightarrow N$ between $g_{n}$ and $g_{n+1}$, whose length $\ell\left(H_{n}\right)$ is bounded by a uniform constant. Define

$$
f(p, t)=H_{n}(p, t-n), \quad t \in[n, n+1] .
$$

We can now prove:

Lemma 5.7. For a geometrically infinite end $e$, the restricted map $\left.f\right|_{M_{e}}$ is liftably quasi-Lipschitz.

Proof. It is sufficient to show that there exist constants $a$ and $b$ such that, for any path $\alpha$ in $S_{e} \times[0, \infty)$ of length $\ell_{M}(\alpha) \leq a$, the image path $f \circ \alpha$ is homotopic with endpoints fixed to a path $\beta$ with length at most $b$.

Thus, let $\alpha:[0,1] \rightarrow S_{e} \times[0, \infty)$ be a path with $\ell_{M}(\alpha) \leq 1$. Decompose $\alpha:[0,1] \rightarrow S_{e} \times[0, \infty)$ as a product $p \times t$, where $p:[0,1] \rightarrow S_{e}$ and $t:[0,1] \rightarrow[0, \infty)$ are the projections of $\alpha$ to the factors. 
By the form of the metric $s$ in (5.3), the projection $S_{e} \times[0, \infty) \rightarrow[0, \infty)$ is non-expanding, and so $t([0,1])$ is contained in some interval $[x, x+1]$. We may assume without loss of generality that $t(0)$ is an integer-if not, we may break $\alpha$ into two parts and argue separately about each, or lengthen $\alpha$ slightly to a path that does begin at an integer point. The projection $S_{e} \times[x, x+$ $1] \rightarrow S_{e} \times\{y\}$ is $\exp (2)$-Lipschitz for any $y \in[x, x+1]$, and thus the length of $p([0,1]) \times r(0)$ is bounded by $\exp (1)$. Since by Lemma $\left.5.6 f\right|_{S_{e} \times t(0)}$ is uniformly liftably quasi-Lipschitz, we conclude that the image of $f \circ(p \times t(0))$ is homotopic rel endpoints to a curve of bounded length.

The image in $N$ of $f \circ(p(1) \times t)$ is contained in adjacent trajectories of $H_{t(0)-1}$ and $H_{t(0)}$, whose length is uniformly bounded. Thus this curve too is homotopic rel endpoints to a curve of bounded length.

Since the path $\alpha^{\prime}$ obtained by concatenating $p \times t(0)$ with $p(1) \times t$ is homotopic, rel endpoints, to $\alpha$, we have shown how to deform $f \circ \alpha$ to a curve of bounded length.

Lifting to a quasi-isometry. To complete the proof of Theorem 5.1, we will show how to apply Corollary 3.2 of Lemma 3.1 to the map $f$.

The first step is to show that $f$ satisfies condition Q1 (cobounded homotopy equivalence). It is obvious from the construction that $f$ is a homotopy equivalence, and we will now prove the following, which implies that $f$ is cobounded (and in fact surjective):

Lemma 5.8. The map $f$ is proper, and of degree 1 .

Proof. First, it is easy to see that $f$ is a proper map. This is immediate from the definition for $\left.f\right|_{M_{e}}$ when $e$ is geometrically finite, and follows when $e$ is simply degenerate from the fact that the pleated surfaces whose induced metrics leave every compact set of $\mathscr{T}\left(S_{e}\right)$ must leave every compact set of $N$, as in the discussion in section 9 (or, more quantitatively, we may apply Lemma 5.9). Thus $f$ defined on all of $M$ is proper. In fact, we know that $f\left(S_{e} \times\{t\}\right)$ is contained eventually in any neighborhood of $e$, as $t \rightarrow \infty$.

Since $f$ is proper, it has a well-defined degree, and we claim that this degree is 1. Let $M(R)$ denote $M-\bigcup_{e}\left(S_{e} \times(R, \infty)\right)$. Then for each $x \in N$ let $d_{R}(x)$ denote the degree with which $\left.f\right|_{M(R)}$ covers $x$ (provided $x \notin f(\partial M(R))$ ). We must show that $d_{R}(x)$ is eventually 1 for all $x$.

Because $N$ is topologically tame, there is a sequence $C_{i} \subset N$ of compact submanifolds whose interiors are homeomorphic to $N$, which eventually contain any compact set in $N$. Further, $N-C_{i}$ is a union of neighborhoods of the ends of the form $N_{e}^{i} \cong S_{e} \times(0, \infty)$. Consider $i$ for which $x \in C_{i}$. Let $R$ be large enough that, for each $e, f$ maps $S_{e} \times(R, \infty) \subset M_{e}$ into the corresponding component $N_{e}^{i}$ of $N-C_{i}$, and $f_{*}$ maps $\pi_{1}\left(S_{e} \times\{R\}\right)$ isomorphically onto $\pi_{1}\left(N_{e}^{i}\right)$ (the second part follows from the fact that, as a consequence of condition $\mathrm{C} 1, \partial K$ is incompressible). Now we may compose $f$ with the retraction of $N$ to $C_{i}$ to obtain a map $f^{\prime}: M(R) \rightarrow C_{i}$ which agrees with $f$ on a neigh- 
borhood of $x$, and which is a homotopy equivalence on the boundary. This implies that $f^{\prime}$ has degree 1 on the boundary, and thus on the interior. We conclude that $d_{R}(x)=1$ for large enough $R$, and thus $f$ has degree 1 .

In order to show that $f$ satisfies Q2 (quasi-isometry), we begin with the following:

Lemma 5.9. $f$ is a quasi-isometry on each $M_{e}$.

Proof. If $e$ is a geometrically finite end, this follows from Lemma 5.3.

Let $e$ be a geometrically infinite end. There is a uniform bound, say $D$, on the diameter of each pleated surface in a neighborhood $N_{e}$ of $e$, and of each cross-section $S_{e} \times\{t\} \subset M_{e}$. The first is a standard fact about hyperbolic surfaces with a lower bound on injectivity radius, and the second follows from this fact, via Lemma 3.3.

Let $(p, r)$ and $(q, t)$ be two points in $M_{e}$, for which we must show that the distance of their images in $N$ is estimated by their distance in $M$. It follows from the expression (5.2) for $d s$ that

$$
|r-t| \leq d_{M}((p, r),(q, t)) \leq|r-t|+D,
$$

and we note that

$$
|r-t|=d_{T}\left(\sigma_{e}(r), \sigma_{e}(t)\right) .
$$

We may assume, without loss of generality, that $r$ and $t$ are integers-replacing them with the nearest integers changes the distances in $\mathscr{T}(S)$ and in $N$ by only a bounded amount. Thus $g_{r}=\left.f\right|_{S_{e} \times\{r\}}$ and $g_{t}=\left.f\right|_{S_{e} \times\{t\}}$ are pleated surfaces, with induced metrics $\rho_{r}$ and $\rho_{t}$ on $S_{e}$. Let $N^{\prime}$ denote the cover of $N$ corresponding to $f_{*} \pi_{1}\left(S_{e}\right)$, and let $g_{r}^{\prime}, g_{t}^{\prime}: S_{e} \rightarrow N^{\prime}$ be the corresponding lifts of $g_{r}, g_{t}$ to $N^{\prime}$. Then Lemmas 4.7 and 4.8 imply

$$
d_{N^{\prime}}\left(g_{r}^{\prime}, g_{t}^{\prime}\right) \simeq d_{T}\left(\sigma_{e}(r), \sigma_{e}(t)\right)
$$

and thus, by (5.4) and (5.5),

$$
d_{N^{\prime}}\left(g_{r}^{\prime}, g_{t}^{\prime}\right) \simeq d_{M}((p, r),(q, t))
$$

where the constants for " $\simeq$ " depend on $\chi(S)$ and $\epsilon$. Therefore (using the diameter bound on images of pleated surfaces) we have

$$
d_{N^{\prime}}\left(f^{\prime}(p, r), f^{\prime}(q, t)\right) \simeq d_{M}((p, r),(q, t))
$$

where $f^{\prime}$ is the lift of $\left.f\right|_{M_{e}}$ to $N^{\prime}$.

Let $N_{e}$ be the component of $N-K$ bounded by $S_{e}$. Then there is a homeomorphic lift $h: N_{e} \rightarrow N^{\prime}$. We claim that, for $x, y$ far enough out in $N_{e}, d_{N^{\prime}}(h(x), h(y))$ and $d_{N}(x, y)$ are equal. Let $(g, \rho)$ be a pleated surface whose image is in $N_{e}$ and such that $d_{N}(g(S), K)>D$, where $D$ is the diameter bound on any pleated surface homotopic to $g$ in $N$. By intersection-number arguments (using the fact that $N_{e}$ is homeomorphic to $S_{e} \times(0, \infty)$ and that $g$ 
is homotopic within $N_{e}$ to the inclusion map of $S_{e}$ ), there is some $R$ such that if $x \in N_{e}$ and $d_{N}(x, K) \geq R$, every path from $x$ to $K$ must hit $g(S)$. Now consider $x, y \in N_{e}$ with $d_{N}(x, K), d_{N}(y, K) \geq R$. Let $\alpha$ be the shortest path in $N$ connecting $x$ to $y$. If $\alpha$ passes through $K$ we can cut it off at $g(S)$ and put in a short cut, obtaining a path of length at most $\ell(\alpha)-D$, which is a contradiction. Thus $d_{N}(x, y)=d_{N_{e}}(x, y)$. Since $h$ is an isometry of $N_{e}$ to its image in $N^{\prime}$, we have $d_{N}(x, y)=d_{N^{\prime}}(h(x), h(y))$.

This implies that $d_{N}(x, y) \simeq d_{N^{\prime}}(h(x), h(y))$ for any $x, y \in N_{e}$. In particular, we may combine this with (5.6) to complete the proof of the lemma.

To prove that $f$ is a global quasi-isometry, we note that by Lemma $5.9 f$ is a quasi-isometry on each of the pieces of $M$ (on the compact piece there is nothing to prove). By topological considerations similar to those made above, there is some $R>0$ such that, if $x \in S_{e} \times[R, \infty)$ and $x^{\prime} \in S_{e^{\prime}} \times[R, \infty)$ for $e \neq e^{\prime}$, then the shortest path from $f(x)$ to $f\left(x^{\prime}\right)$ must pass through $f\left(S_{e} \times\{0\}\right)$ and $f\left(S_{e^{\prime}} \times\{0\}\right)$. Thus

$$
\begin{aligned}
d_{N}\left(f(x), f\left(x^{\prime}\right)\right) & \simeq d_{N}\left(f(x), f\left(\partial M_{e}\right)\right)+d_{N}\left(f\left(x^{\prime}\right), f\left(\partial M_{e^{\prime}}\right)\right) \\
& \simeq d_{M}\left(x, \partial M_{e}\right)+d_{M}\left(x^{\prime}, \partial M_{e^{\prime}}\right) \\
& \simeq d_{M}\left(x, x^{\prime}\right)
\end{aligned}
$$

so that $f$ is a quasi-isometry.

Condition $\mathrm{Q} 3$, that $f$ is liftably quasi-Lipschitz, holds globally if it holds for each piece, and this is the content of Lemmas 5.3 and 5.7. (On the compact piece $K, f$ is Lipschitz by construction.)

It remains to prove condition $\mathrm{Q}^{\prime}$ of Corollary 3.2-that loops of bounded length in $N$ can be deformed (with basepoints fixed) to images of loops of bounded length in $M$.

Fix any $B>0$, and let $e$ be a simply degenerate end of $N$. By Lemma 5.9, there is some $t_{0}$ so that $f\left(S_{e} \times\left[t_{0}, \infty\right)\right)$ is contained in the product neighborhood $N_{e}$ of $e$, and does not meet any point closer than $B / 2$ to its boundary. Consider now a point $(p, t) \in S_{e} \times\left[t_{0}, \infty\right)$, and let $\beta$ be a loop based at $f(p, t)$ of $N$-length at most $B$. By the choice of $t_{0}, \beta$ is completely contained in $N_{e}$. Since $\pi_{1}\left(S_{e}\right)$ is mapped isomorphically to $\pi_{1}\left(N_{e}\right)$, there is a loop $\alpha \subset S_{e}$ through $p$ such that $f(\alpha \times\{t\})$. is homotopic to $\beta$ (keeping the basepoint fixed). Again let us assume that $t$ is an integer, possibly adding a bounded-length section to $\alpha \times\{t\}$. Then $f$ restricted to $S_{e} \times\{t\}$ is a pleated surface $g_{t}$. If we take $\alpha$ to be of minimal $\rho_{t}$-length in the homotopy class with $p$ fixed, and apply Lemma 4.4 , we obtain a uniform bound on $\ell_{\rho_{t}}(\alpha)$. This yields a bound on $\ell_{\sigma_{e}(t)}(\alpha)$, since, as before, the identity on $S_{e}$ is a liftably quasi-isometric map (with uniform constants) between $\sigma_{e}(t)$ and $\rho_{e}(t)$. We conclude that condition $\mathrm{Q}^{\prime}$ holds when the basepoint lies in $S_{e} \times\left[t_{0}, \infty\right)$.

For a geometrically finite end the condition is vacuously satisfied: Fixing $B$, there is a compact subset of $M_{e}$ and of the corresponding neighborhood of $e$ 
in $N$, outside of which there are no loops of length less than $B$. The condition clearly holds in the remaining portion of $M$, which is compact. Thus $\mathrm{Q}^{\prime}$ holds everywhere for $f$.

Since Q1-3 and Q4 ${ }^{\prime}$ are satisfied, Corollary 3.2 of Lemma 3.1 implies that $\tilde{f}$ is a cobounded (in fact surjective) quasi-isometry.

The model metric for surface groups. In the case where $N \cong S \times \mathbf{R}$, we can adjust the construction a little so that the model manifold takes on a particularly nice form, which will be used in sections 7 and 8 . Consider first the case where both ends are simply degenerate, and let $\lambda_{+}, \lambda_{-}$denote the two ending laminations. In this case. we claim, there exists a conformal structure $\sigma$ on $S$ and a quadratic differential $\Phi$, holomorphic with respect to $\sigma$, so that $\lambda_{+}$and $\lambda_{-}$are equivalent to the foliations $\Phi_{v}$ and $\Phi_{h}$. Note first that, as mentioned before the statement of Theorem 5.5, $\lambda_{+}$and $\lambda_{-}$are uniquely ergodic, so the measure class of the equivalent foliations is determined. Gardiner-Masur [GM91] and Kerckhoff [Ker92] showed that a pair of measured laminations (or foliations) are obtained from a holomorphic quadratic differential provided that they fill up $S$ (see section 2). In our case, Lemma 2.4 implies that $\lambda_{+}$and

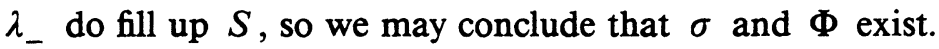

The pair $\sigma$ and $\Phi$ determine a Teichmüller geodesic $L$ passing through $[\sigma]$, which can be divided into two rays, $R(\sigma, \Phi)$ terminating in $\Phi_{v}$, and $R(\sigma,-\Phi)$ terminating in $\Phi_{h}$. The conclusions of Theorem 5.5 (Ending ray) hold for these rays, since we may select $\sigma$ as our basepoint. Since in this case $M \sim S \times \mathbf{R}$, we may dispense with the compact core $K \cong S \times[0,1]$ and simply define the model metric as follows:

$$
d s^{2}=e^{2 t} d x^{2}+e^{-2 t} d y^{2}+d t^{2}
$$

where $d x$ and $d y$ are the transverse measures corresponding to $\Phi$, as before, and $t$ ranges over $(-\infty, \infty)$.

If $N$ has just one simply degenerate end, to which we associate a ray $R(\sigma, \Phi)$, we may write a metric on $M$ as follows:

$$
d s^{2}=e^{|2 t|} d x^{2}+e^{-2 t} d y^{2}+d t^{2}
$$

for $t \in(-\infty, \infty)$. For positive $t$, this is equal to the metric in Theorem 5.1 (The model manifold). For (sufficiently large) negative $t$ the metric on a slice $S \times\{t\}$ in the theorem was taken as a dilation of a hyperbolic metric on $S$ by $(\cosh t)^{2}$, whereas here it is a dilation by $e^{|2 t|}$ of a quadratic differential metric. By Lemma 3.3, the two metrics on $S$ are liftably quasi-isometric. Therefore:

Corollary 5.10. If $N \cong S \times \mathbf{R}$ then the metric described by (5.7) when $N$ has two degenerate ends, and by (5.8) when $N$ has one degenerate end, is liftably quasi-isometric to the model metric constructed in Theorem 5.1. In particular the conclusions of Theorem 5.1 hold for this metric as well. 


\section{THE ENDING LAMINATION THEOREM}

The proof of the main theorem is now a brief application of the foregoing constructions.

Theorem A (Ending lamination theorem). Let $N_{1}$ and $N_{2}$ be two homeomorphic hyperbolic manifolds satifying C1-2. If corresponding ends of $N_{1}$ and $N_{2}$ have equal end invariants, then the homeomorphism is homotopic to an isometry.

Proof. Let $G=\pi_{1}\left(N_{1}\right)=\pi_{1}\left(N_{2}\right)$. Let $\rho_{i}: G \rightarrow \operatorname{PSL}_{2}(\mathrm{C})$ be a representation such that $\mathbf{H}^{3} / \rho_{i}(G)$ induces the given hyperbolic structure on $N_{i}$.

Theorem 5.1 (The model manifold) gives us two quasi-isometries $f_{i}: M \rightarrow$ $N_{i}$ which lift to cobounded quasi-isometries $\tilde{f}_{i}$. Let $\tilde{f}_{1}^{-1}$ denote a quasi-inverse of $\tilde{f}_{1}$. Then, letting $F=\tilde{f}_{2} \circ \tilde{f}_{1}^{-1}$, we can check that the following holds:

$$
d\left(F\left(\rho_{1}(g)(x)\right), \rho_{2}(g)(F(x))\right) \leq A
$$

for some constant $A$, and all $x \in \mathbf{H}^{3}, g \in G$. That is, $F$ approximately conjugates the action of $\rho_{1}(G)$ to that of $\rho_{2}(G)$.

By Theorem 3.7, $F$ extends to a quasiconformal homeomorphism $\mathscr{F}: \hat{\mathbf{C}} \rightarrow$ $\hat{\mathbf{C}}$. It then follows immediately that $\mathscr{F}$ exactly conjugates the actions of $\rho_{1}(G)$ and $\rho_{2}(G)$ on $\hat{\mathbf{C}}$.

Furthermore, $\mathscr{F}$ is conformal on the domain of discontinuity $\Omega_{\rho_{1}(G)}$, by virtue of Lemma 5.4: Each $f_{i}$, restricted to a neighborhood of a geometrically finite end $e$, can be represented as a map of an end of a Fuchsian manifold $\mathbf{H}^{3} / \Gamma_{0}$ which, lifted to the universal cover, extends to a conformal map of a component of $\Omega_{\Gamma_{0}}$ to the corresponding component of $\Omega_{\rho_{i}(G)}$. It follows that the composition is conformal as required.

We can now apply Sullivan's rigidity theorem:

Theorem 6.1 (Sullivan [Sul81]). Let $\Gamma_{1}, \Gamma_{2}$ be two finitely generated Kleinian groups acting on $\hat{\mathbf{C}}$, and $\mathscr{F}: \hat{\mathbf{C}} \rightarrow \hat{\mathbf{C}}$ a quasiconformal homeomorphism such that

$$
\Gamma_{2}=\mathscr{F}^{\circ} \Gamma_{1} \circ \mathscr{F}^{-1}
$$

and $\mathscr{F}$ is conformal on the domain of discontinuity of $\Gamma_{1}$. Then $\mathscr{F}$ is a Möbius transformation.

We conclude that our map $\mathscr{F}$ is a Möbius transformation, and thus the quotient manifolds are isometric.

\section{The Cannon-Thurston theorems}

In [CT89], Cannon and Thurston proved a theorem that characterizes the topological structure of the action of certain degenerate surface groups on their limit sets. In particular, they showed that these limit sets are continuously parametrized by the circle in a natural way, so that in those cases where the limit 
set is the entire sphere $\hat{\mathbf{C}}$, a group-equivariantly parametrized Peano curve is obtained. In this section we shall extend their results to all surface groups which satisfy our injectivity radius condition (see also Fenley [Fen92] for a different generalization of the Cannon-Thurston theorems).

The first result may be stated as follows.

Theorem B (Peano curves). Let $\Gamma=\rho\left(\pi_{1}(S)\right)$ be a Kleinian group satisfying $\mathrm{C} 2$, where $S$ is a closed surface, and let $\rho_{0}$ be a Fuchsian representation of $\pi_{1}(S)$, with limit set $\Lambda_{0} \cong S^{1}$. Then there is a continuous map $\phi: S^{1} \rightarrow \Lambda_{\Gamma}$, which takes the action by $\rho_{0}$ on $S^{1}$ to the action of $\rho$ on $\Lambda_{\Gamma}$.

An immediate consequence of Theorem $B$ is the following, which follows directly from the fact that $\Lambda_{\Gamma}$ is the continuous image of a circle.

Corollary C. If $\Gamma$ is a surface group satisfying $\mathrm{C} 2$, then $\Lambda_{\Gamma}$ is locally connected.

The groups for which Theorem B is proved in [CT89] are of two types. First, there are groups $\Gamma \cong \pi_{1}(S)$ obtained as the fiber group of a compact manifold that fibers over the circle. That is, if $\varphi: S \rightarrow S$ is a pseudo-Anosov homeomorphism and $N_{\varphi}=S \times[0,1] / \varphi$ is the mapping torus obtained by identifying $S \times\{0\}$ with $S \times\{1\}$ via $\varphi$, then Thurston showed in [Thua] that $N_{\varphi}$ admits a hyperbolic structure. The infinite cyclic cover $\hat{N}_{\varphi}$ has fundamental group $\pi_{1}(S)$, and has two simply degenerate ends. It is not hard to see, since this manifold admits a cocompact $\mathbf{Z}$ action which induces $\varphi_{*}$ on the fundamental group, that Theorem 5.1 holds here (without needing our proof), where the geodesic $L$ is the axis of the action of $\varphi_{*}$ on $\mathscr{T}(S)$.

The mapping torus $\hat{N}_{\varphi}$ is obtained as a limit of a subsequence of quasiFuchsian groups $\left\{q f\left(\varphi_{*}^{-n} \sigma, \varphi_{*}^{n} \sigma\right)\right\}$, where $[\sigma] \in \mathscr{T}(S)$ is a fixed point and $q f(x, y)$ is a quasi-Fuchsian group uniformizing $x$ and $y$ (see [Ber60, Thua]). If instead we consider $\left\{q f\left(\sigma, \varphi_{*}^{n} \sigma\right)\right\}$, there is again a convergent subsequence, and the limit manifold has one geometrically finite end, and one simply degenerate end. This is the second type of group to which the proof in [CT89] applies.

One might also think of Theorem B in the following terms: $\pi_{1}(S)$, viewed as an abstract group, is "negatively curved" in the sense of Cannon [Can91] or "hyperbolic" in the sense of Gromov [Gro87, CDP90, GdlH90]. Its ideal boundary $\partial \pi_{1}(S)$ is just $S^{1}$, and is naturally identified with the limit set of the Fuchsian representation [Flo80]. Thus it is not hard to see that Theorem B implies that the limit set $\Lambda_{\Gamma}$ is naturally parametrized by $\partial \Gamma$.

If $\Gamma$ is any Kleinian group whose quotient satisfies $C 1-2$, then $\Gamma$ is certainly Gromov-hyperbolic, because $\Gamma$ also admits a convex-cocompact representation, by Thurston's geometrization theorem. Let $\Gamma_{0}$ denote the image of this representation. Again, the ideal boundary $\partial \Gamma$ is naturally homeomorphic to $\Lambda_{\Gamma_{0}}$ (see [Can91]). It is natural to expect Theorem B to generalize to a statement 


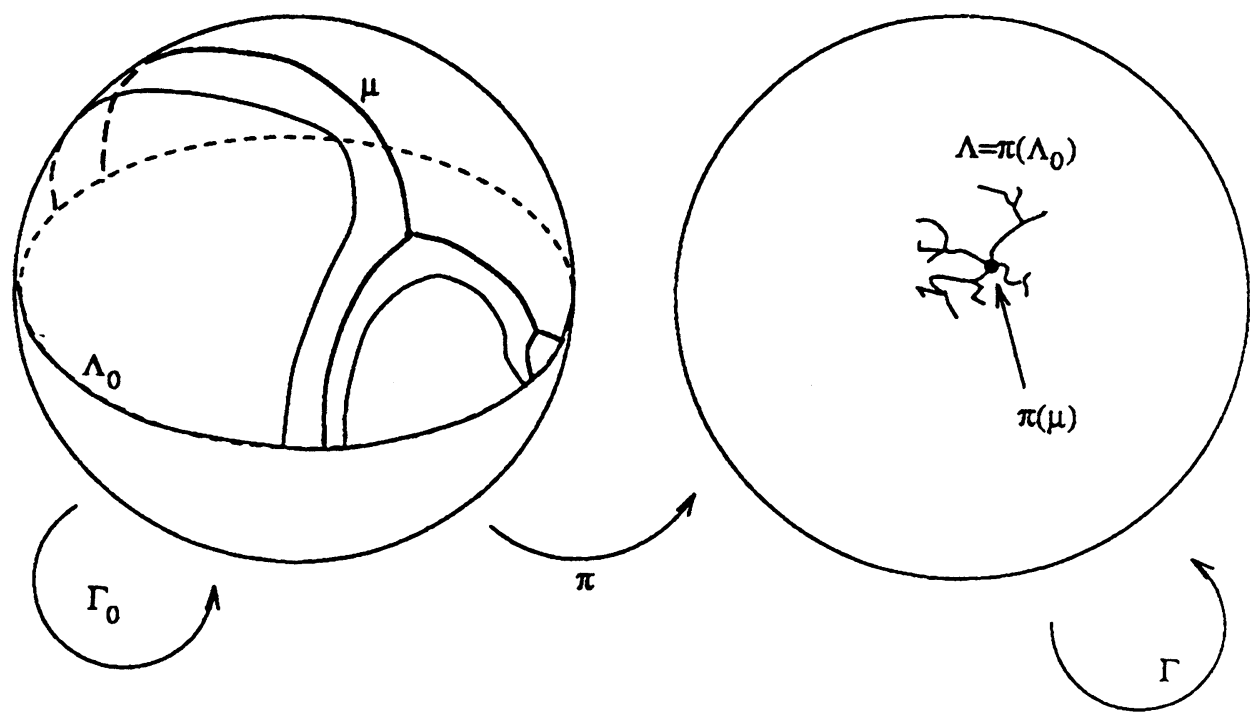

FIGURE 3. A sketch of the map $\pi: \hat{\mathbf{C}} \rightarrow \hat{\mathbf{C}}$, taking a leaf $\mu$ of $\widetilde{\Phi}_{+}$to a point, and the action of $\Gamma_{0}$ to that of $\Gamma$. Pictured is the case where $\Gamma$ has one simply degnerate end.

about a collapsing map semi-conjugating $\Gamma_{0}$ to $\Gamma$, and in particular one can make the following well-known conjecture:

Conjecture 7.1. Suppose $N=\mathbf{H}^{3} / \Gamma$ satisfies $\mathrm{C1-2}$. Then there is a map $\varphi$ : $\partial \Gamma \rightarrow \Lambda_{\Gamma}$ which is continuous, and equivariant with respect to the action of $\Gamma$.

Theorem B is a consequence of the next theorem, which gives a stronger topological characterization of the action of $\Gamma$ on the sphere. This again is an extension of a theorem which was proven in [CT89] for the cases arising, as above, from pseudo-Anosov homeomorphisms.

Let $\Gamma=\rho\left(\pi_{1}(S)\right)$ be any representation of $\pi_{1}(S)$ satisfying C2, which has at least one simply degenerate end, and let $\Gamma_{0}=\rho_{0}\left(\pi_{1}(S)\right)$ be a fixed Fuchsian representation. We shall describe a continuous map $\pi: \hat{\mathbf{C}} \rightarrow \hat{\mathbf{C}}$ which semiconjugates the action of $\Gamma_{0}$ to that of $\Gamma$. That is,

$$
\pi \circ \rho_{0}(\gamma)=\rho(\gamma) \circ \pi
$$

for any $\gamma \in \pi_{1}(S)$. In particular, it follows that $\Lambda_{\Gamma}$ is the continuous image of $\Lambda_{\Gamma_{0}}$, which is a circle, and thus Theorem B holds (see Figure 3).

Furthermore, the collapse is determined by the ending lamination(s) of $\Gamma$. Let $\lambda_{+}, \lambda_{-}$be the ending laminations of the simply degenerate ends of $\Gamma$, if there are two, or let $\lambda_{+}$denote the one ending lamination if $\Gamma$ has one degenerate end (we henceforth refer by $\lambda_{ \pm}$to the laminations in either case). Let $\Omega_{+}$ and $\Omega_{-}$denote the corresponding components of the domain of discontinuity 
of $\Gamma_{0}$. We can represent $\lambda_{ \pm}$as foliations $\Phi_{ \pm}$, which we lift to $\widetilde{\Phi}_{ \pm}$on $\Omega_{ \pm}$. The theorem is now:

Theorem $\mathbf{D}$ (Topology of the action). If $\Gamma=\rho\left(\pi_{1}(S)\right)$ is a surface group satisfying $\mathrm{C} 2$ then there is a continuous map $\pi: \hat{\mathbf{C}} \rightarrow \hat{\mathbf{C}}$ such that (7.1) holds, and $\pi$ collapses the leaves of $\widetilde{\Phi}_{ \pm}$to points. That is, if $\Gamma$ has one degenerate end then $\pi(x)=\pi(y)$ if and only if $x$ and $y$ lie on the closure of the same leaf of $\widetilde{\Phi}_{+}$. If both ends of $\Gamma$ are degenerate then $\pi(x)=\pi(y)$ also if $x$ and $y$ lie on the closure of the same leaf of $\widetilde{\boldsymbol{\Phi}}_{-}$. If $\Gamma$ is quasi-Fuchsian then $\pi$ is a homeomorphism.

Note. In the quasi-Fuchsian case there exists, by definition, such a map $\pi$ which is a quasiconformal homeomorphism.

Proof. The proof follows the argument of [CT89], but there are some changes in both substance and presentation.

By Corollary 5.10, the metric $d s$ on $M=S \times \mathbf{R}$ and its universal cover $\widetilde{S} \times \mathbf{R}$ can be expressed by the formula (5.7) when $N$ has two degenerate ends, and by (5.8) when there is one degenerate end. Thus in particular there is a quadratic differential $\Phi$ on a fixed surface $(S, \sigma)$ such that $\Phi_{v}$ corresponds to $\lambda_{+}$and (in the case of two degenerate ends) $\Phi_{h}$ is $\lambda_{-}$. The metric $d s$ restricted to $S \times\{0\}$ is $|\Phi|$.

We may identify $\mathbf{H}^{3}$ with $\mathbf{H}^{2} \times \mathbf{R}$ as follows: Let $\mathbf{H}^{2} \subset \mathbf{H}^{3}$ be the geodesic plane preserved by the Fuchsian group $\Gamma_{0}$. For $x \in \mathbf{H}^{3}$ let $p(x)$ denote the nearest point in $\mathbf{H}^{2}$ to $x$, and let $t(x)$ denote the signed distance to $\mathbf{H}^{2}$. Then $x \mapsto(p(x), t(x))$ is the desired identification, and in these coordinates we may express the hyperbolic metric as

$$
d h^{2}=(\cosh t)^{2} d p^{2}+d t^{2}
$$

where $d p^{2}$ is the hyperbolic metric on $\mathbf{H}^{2}$.

Fix an identification $\psi: \mathbf{H}^{2} \rightarrow \widetilde{S}$ which respects the identification $\rho_{0}^{-1}$ : $\Gamma_{0} \rightarrow \pi_{1}(S)$. This extends uniquely to a homeomorphism

$$
\psi: \mathbf{H}^{3} \rightarrow \widetilde{M}=\widetilde{S} \times \mathbf{R}
$$

which respects the product structures, and the group actions. We shall fix $\psi$ for the remainder of the proof. Now if $\widetilde{f}$ is the map given by Theorem 5.1, we can define

$$
F=\tilde{f} \circ \psi: \mathbf{H}^{3} \rightarrow \mathbf{H}^{3}
$$

which conjugates the action on $\mathbf{H}^{3}$ of $\Gamma_{0}=\rho_{0}\left(\pi_{1}(S)\right)$ to that of $\Gamma=\rho\left(\pi_{1}(S)\right)$.

To prove the theorem it will suffice to show that $F$ extends to a map of $\mathbf{H}^{3} \cup \hat{\mathbf{C}}$ to $\mathbf{H}^{3} \cup \hat{\mathbf{C}}$ which is continuous, and collapses leaves of the foliations as in the statement of the theorem. 
Geometry of the foliation leaves. We begin with a discussion of the geometry of leaves of $\widetilde{\Phi}_{h}$ and $\widetilde{\Phi}_{v}$ under the metric $|\widetilde{\Phi}|$ on the plane $\widetilde{S}$ (see [Str84, HM79]). By a full leaf of $\widetilde{\Phi}_{h}$ or $\widetilde{\Phi}_{v}$ we shall mean a component of either one of the two singular foliations; in particular a full leaf may be a tree if it contains singularities. By a leaf we mean the boundary of a component of the complement of a full leaf (so a leaf is topologically a line, obtained as a subset of a full leaf).

Note first that $|\widetilde{\Phi}|$ is a non-positively curved complete metric on $\widetilde{S}$-in fact it is Euclidean except for the singularities where negative curvature is concentrated. We have the following well-known consequences of this:

(1) Leaves of $\widetilde{\Phi}_{h}$ and $\widetilde{\Phi}_{v}$ are $|\widetilde{\Phi}|$-geodesics, and more generally a $|\widetilde{\Phi}|$ geodesic is any path which is Euclidean away from singularities, and makes angles of no less than $\pi$ on both sides where it meets a singularity.

(2) Between any two points $p, q$ in $\widetilde{S}$ there is a unique $|\widetilde{\Phi}|$-geodesic segment, which we write $[p, q]$.

(3) Any $|\widetilde{\Phi}|$-geodesic $L$ admits a well-defined nearest-point retraction $\pi_{L}$ : $\mathbf{H}^{2} \rightarrow L$, which is non-increasing in the $|\widetilde{\Phi}|$ metric. Furthermore $\pi_{L}$ strictly contracts the length of any path between points in $L$ which is not contained in $L$.

Furthermore, for an arc $\alpha$ in $\tilde{S}$ we can define intersection numbers $i\left(\alpha, \widetilde{\Phi}_{v}\right)=$ $\int_{\alpha}|d x|$ and $i\left(\alpha, \widetilde{\Phi}_{h}\right)=\int_{\alpha}|d y|$, and we have (see [HM79, §3])

(4) The geodesic $[p, q]$ minimizes intersection number with either foliation, over all curves joining $p$ to $q$.

Totally geodesic planes. Let $L$ be a leaf of $\widetilde{\Phi}_{h}$ or $\widetilde{\Phi}_{v}$. Then $L \times \mathbf{R}$ is a plane in $\tilde{S} \times \mathbf{R}$, whose geometry is characterized by the following:

Lemma 7.2. If $L$ is a leaf of $\widetilde{\boldsymbol{\Phi}}_{v}$ or $\widetilde{\boldsymbol{\Phi}}_{h}$ then

(1) The plane $L \times \mathbf{R}$ is totally geodesic in $\tilde{S} \times \mathbf{R}$ with respect to $s$.

(2) The restriction of the metric $s$ to $L \times \mathbf{R}$ renders it quasi-isometric to the hyperbolic plane. In fact if $L$ is a leaf of $\Phi_{v}$ or if $s$ is given by (5.7), then $(L \times \mathbf{R}, s)$ is exactly hyperbolic.

(3) The image $\psi^{-1}(L \times \mathbf{R})$ is quasiconvex in $\mathbf{H}^{3}$.

(4) The hyperbolic metric $h$ restricted to $\psi^{-1}(L \times \mathbf{R})$ renders it isometric to the hyperbolic plane.

Proof. Part (1): Extend $\pi_{L}$ to a map $\pi: \tilde{S} \times \mathbf{R} \rightarrow L \times \mathbf{R}$ via $\pi(p, t)=$ $\left(\pi_{L}(p), t\right)$. Since $\pi_{L}$ is distance-non-increasing and $\pi$ leaves the $t$ direction invariant, it follows that $\pi$ is distance-non-increasing. In fact since paths not in $L$ with endpoints in $L$ are strictly contracted by $\pi_{L}$, it follows easily that $L \times \mathbf{R}$ is 0 -quasiconvex, or totally geodesic.

Part (2) follows immediately from the formulas (5.7) and (5.8) for $d s$. In fact one can easily see that the metric $e^{-2 t} d x^{2}+d t^{2}$ is exactly the hyperbolic 
metric, under a parametrization where the lines $\{t=c\}$ are horocycles and $\{x=c\}$ are orthogonal geodesics. The metric $e^{|2 t|} d x^{2}+d t^{2}$ is quasi-isometric to $(\cosh t)^{2} d x^{2}+d t^{2}$, which is the hyperbolic metric in a parametrization where $\{t=0\}$ is a geodesic, $\{t=c\}$ are equidistant curves to it, and $\{x=c\}$ are again orthogonal geodesics.

For part (3), notice that $L^{\prime}=\psi^{-1}(L)$ is a hyperbolic quasigeodesic in $\mathbf{H}^{2} \subset$ $\mathbf{H}^{3}$, since the identification between $\mathbf{H}^{2} / \Gamma_{0}$ and $(S,|\Phi|)$ can be chosen to be Lipschitz (the surfaces being compact). The plane $\psi^{-1}(L \times \mathbf{R})$ can be written as $P=p^{-1}\left(L^{\prime}\right)$ where $p: \mathbf{H}^{3} \rightarrow \mathbf{H}^{2}$ is the projection discussed earlier. Now, for any two points $x, y \in P$ it is an easy exercise in hyperbolic geometry to see that the hyperbolic geodesic segment $[x y]$ is contained in a $\delta$-neighborhood of $[x p(x)] \cup[p(x) p(y)] \cup[y p(y)]$, where $\delta$ is a fixed constant depending only on $\mathbf{H}^{3}$. (This is most easily seen as a consequence of Gromov's " $\delta$-hyperbolicity" criterion.) Since $[x p(x)]$ and $[y p(y)]$ are contained in $P$ and $[p(x) p(y)]$ is contained in a uniform neighborhood of the quasigeodesic $L^{\prime}$ (see Theorem 3.5), it follows that $P$ is quasiconvex.

Part (4) follows immediately from the restriction of the metric in (7.2) to a path-metric on $P$ (note in particular that $P$ is a hyperbolic ruled surface).

Halfspaces. Any plane $L \times \mathbf{R}$ divides $\widetilde{S} \times \mathbf{R}$ into two convex halfspaces, $H_{1}$ and $H_{2}$. Let us consider their images $\widetilde{f}\left(H_{i}\right)$, which are quasiconvex by Theorem 3.5. First, we note that $\partial_{\infty}(\tilde{f}(L \times \mathbf{R})) \subset \hat{\mathbf{C}}$ is a Jordan curve $\beta$, since $L \times \mathbf{R}$ is quasi-isometric to $\mathbf{H}^{2}$, and by Theorem 3.6 , the quasi-isometry $\tilde{f}$ restricted to $L \times \mathbf{R}$ extends to infinity. Let $D_{i}=\partial_{\infty} \widetilde{f}\left(H_{i}\right)$, for $i=1,2$. Then it is an easy exercise to show that $D_{1}$ and $D_{2}$ are closed disks, $D_{1} \cap D_{2}=\beta$, and $\hat{\mathbf{C}}=D_{1} \cup D_{2}$.

It will be useful in the next subsection to control the diameter, in the Poincare model of $\mathbf{H}^{3}$, of the images $\widetilde{f}\left(H_{i}\right)$ of these halfspaces. For the following

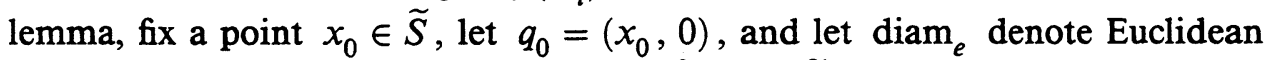
diameter in the Poincare ball model of $\mathbf{H}^{3}$ with $\tilde{f}\left(q_{0}\right)$ taken as the origin.

Lemma 7.3. Let $L$ be a leaf of $\widetilde{\boldsymbol{\Phi}}_{v}$ or $\widetilde{\boldsymbol{\Phi}}_{h}$ such that $d_{|\tilde{\mid}|}\left(x_{0}, L\right)>R$. Let $H$ be the halfspace bounded by $L \times \mathbf{R}$ which does not contain $q_{0}$. Then

$$
\operatorname{diam}_{e}(\tilde{f}(H)) \leq a R^{-\alpha}
$$

where $a, \alpha>0$ depend only on the quasi-isometry constants $K, \delta$ of $\tilde{f}$.

Proof. The idea is that a convex set in $\mathbf{H}^{3}$ far from the origin has small Euclidean diameter in the Poincare model. In fact, it is easy to show that, if $\gamma \subset \mathbf{H}^{3}$ is a geodesic segment such that $d(0, \gamma)>r$, then

$$
\operatorname{diam}_{e}(\gamma) \leq 2 / \sinh r
$$

(see [Bea83], for example). 
We first show that $d_{s}\left(q_{0}, L \times \mathbf{R}\right)$ is large. Let $D=\frac{1}{2} \log R$, and note that inside $\widetilde{S} \times[-D, D]$ we have (consulting the formula for $d s$ ) $|d s| \geq e^{-D}|\tilde{\Phi}|$. Now consider $w=(y, t) \in L \times \mathbf{R}$. If $|t| \leq D / 2$ then, since a path from $w$ to $q_{0}$ must either remain in the set $\tilde{S} \times[-D, D]$ or travel to its boundary and back, we have

$$
d_{s}\left(q_{0}, w\right) \geq \min \left\{D, e^{-D} R\right\} .
$$

If $|t| \geq D / 2$ then $d_{s}\left(q_{0}, w\right) \geq D / 2$. Finally, any point in $H$ is at least as far from $q_{0}$ as $L \times \mathbf{R}$. Thus we conclude

$$
d_{s}\left(q_{0}, H\right) \geq R^{\prime}=\min \left\{\frac{1}{4} \log R, \sqrt{R}\right\} .
$$

Applying the quasi-isometry $\widetilde{f}$, and using the fact that $\widetilde{f}(H)$ is quasiconvex, we may conclude that for any geodesic segment $\gamma$ connecting two points in $\tilde{f}(H)$, $d(0, \gamma) \geq c R^{\prime}-d$, where $c, d>0$ depend on the quasi-isometry constants. Finally applying (7.5), we obtain the desired statement.

Defining the extension of $F$. Lemma 7.2 enables us to understand the extension to infinity of $F$ restricted to one of the planes $P=\psi^{-1}(L \times \mathbf{R})$. Since both $P$ and $L \times \mathbf{R}$ are isometric to $\mathbf{H}^{2}$ (or in one case quasi-isometric), both are naturally compactified by the circle, which we write as $\partial P$ and $\partial(L \times \mathbf{R})$, respectively. We claim that the map $\psi: P \rightarrow L \times \mathbf{R}$ extends continuously to a map $\psi: \partial P \rightarrow \partial(L \times \mathbf{R})$. This can be seen explicitly from the formulas (7.2) for $h$ on $P$ and (5.7) or (5.8) for $d s$ on $L \times \mathbf{R}$. In case $L$ is a leaf of $\widetilde{\Phi}_{v}$, the map $\left.\psi\right|_{P}$ takes the geodesic $\{t=0\}$ on $P$ and its equidistant curves to nested horocycles in $(L \times \mathbf{R})$. This is illustrated in Figure 4. It is easy to see that $\psi$ collapses the upper semicircle of $P$ to a point, and is injective on the lower semicircle. The same is true for $L$ in $\widetilde{\Phi}_{h}$ in case $d s$ is of the form (5.7), except that the lower semicircle is mapped to a point. In the remaining case, where $L$ is a leaf of $\widetilde{\Phi}_{h}$ but $d s$ takes the form (5.8), $\left.\psi\right|_{\partial P}$ is a homeomorphism.

Applying Theorem 3.6 to $\left.\tilde{f}\right|_{L \times \mathbf{R}}$, we conclude that $\left.F\right|_{P \cup \partial P}: P \cup \partial P \rightarrow \mathbf{H}^{3} \cup \hat{\mathbf{C}}$ has exactly the desired identifications on the boundary.

The following lemma enables us to extend $F$ globally to all of $\hat{\mathbf{C}}$.

Lemma 7.4. If $\gamma: \mathbf{R}_{+} \rightarrow \mathbf{H}^{3}$ is a geodesic ray, then the image $\{F(\gamma(t))\}$ has a unique endpoint in $\dot{\hat{\mathbf{C}}}$, which we call $F(\gamma(\infty))$.

Proof. Denoting by $\gamma(\infty)$ the endpoint of $\gamma$ on $\hat{\mathbf{C}}$, consider first the case where $\gamma(\infty)$ lies in the complement $\Omega_{0}$ of the circle $\Lambda_{\Gamma_{0}}$. Then $\gamma$ must be asymptotic to a geodesic ray of the form $\gamma_{q}(t)=(q, \pm t)$ where $q \in \mathbf{H}^{2}$ and we are using the decomposition of $\mathbf{H}^{3}$ as $\mathbf{H}^{2} \times \mathbf{R}$. By Lemma 7.2 and the discussion that follows, $F\left(\gamma_{q}(t)\right)$ is a quasigeodesic and by Theorem 3.6 has a unique endpoint $\xi \in \hat{\mathbf{C}}$. Furthermore, the map $F$ is quasi-Lipschitz, since $\psi$ is Lipschitz (compare the formula for $d h$ with that for $d s$ ) and $\widetilde{f}$ is a quasi-isometry. Thus, $F(\gamma(t))$ must terminate in $\xi$ as well. 

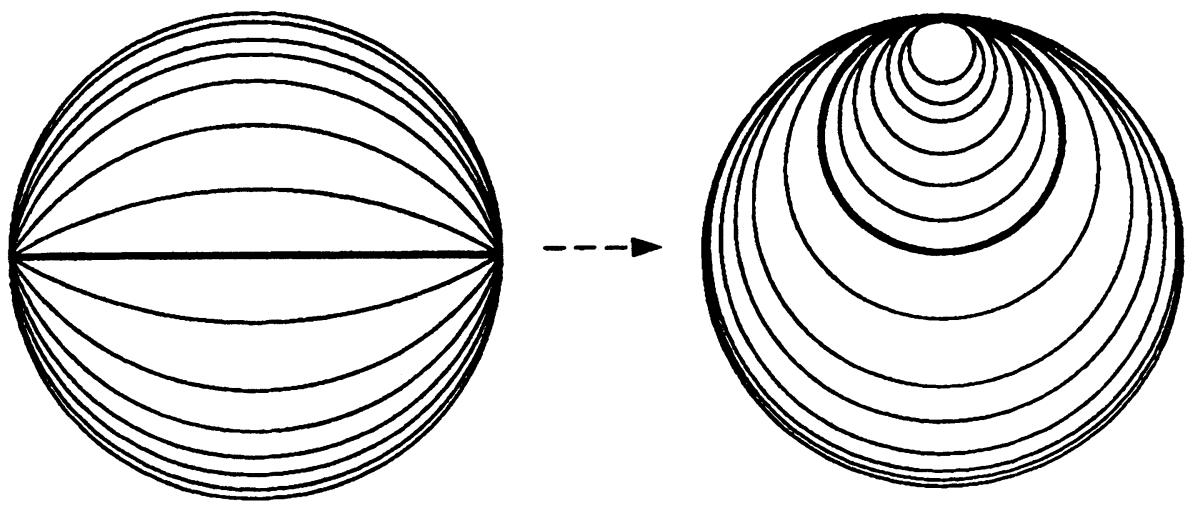

Figure 4. A map of $\mathbf{H}^{2}$ to itself that collapses a half-plane to a horodisk. Each level set of the central geodesic in the left-hand plane is mapped to the corresponding level set of the central horocycle on the right. This map is equivalent to $\psi: \psi^{-1}(L \times \mathbf{R}) \rightarrow(L \times \mathbf{R})$, for an arc $L$ of $\widetilde{\Phi}_{v}$.

For the remaining cases, we will show how to contain the end of a ray $\gamma$ in a nested collection of halfspaces whose images under $F$ will converge to a unique point in $\hat{\mathbf{C}}$.

It will be helpful first to consider the explicit connection between a foliation and its equivalent lamination (see also [Lev83]). Let $\ell=\psi^{-1}(L)$, where $L$ is a leaf of $\widetilde{\Phi}_{v}$. Then $\ell$ is a hyperbolic quasigeodesic, and in particular it meets infinity at two points, and lies in a bounded neighborhood of the hyperbolic geodesic $\ell^{*}$ connecting those two points. The union of geodesics $\ell^{*}$ forms a lamination $\tilde{\lambda}_{v}$ which projects down to the lamination $\lambda_{v}$ on $S$ that corresponds to $\Phi_{v}$. The same construction works for $\widetilde{\Phi}_{h}$.

Now consider a geodesic ray $\gamma$ in $\mathbf{H}^{3}$ with $\gamma(\infty) \in \Lambda_{\Gamma_{0}}$, and let $\gamma^{\prime}$ denote a geodesic ray in $\mathbf{H}^{2}$ asymptotic to $\gamma$. We claim that $\psi \circ \gamma^{\prime}$ cuts through a sequence of leaves $L_{i}$ of $\widetilde{\boldsymbol{\Phi}}_{h}$ or $\widetilde{\boldsymbol{\Phi}}_{v}$ that leave every compact set.

By Lemma $2.4, \lambda_{h}$ and $\lambda_{v}$ fill up $S$, so that their lifts $\tilde{\lambda}_{h}$ and $\tilde{\lambda}_{v}$ cut $\mathbf{H}^{2}$ into a collection of bounded disks. Thus $\gamma^{\prime}$ must intersect a sequence of leaves $\ell_{i}^{*}$ of these laminations, such that the intersection points leave every compact set. Suppose that the leaves themselves continue to intersect a compact set. Then a subsequence converges to a leaf $\ell^{*}$ which is contained in one of the laminations, say $\tilde{\lambda}_{h}$, and is asymptotic to $\gamma^{\prime}$. But then, by the same reasoning, $\ell^{*}$ must hit infinitely many leaves $\ell_{i}^{\prime}$ in $\tilde{\lambda}_{v}$. If these intersect a fixed compact set we obtain a limit leaf $\ell^{\prime}$ in $\tilde{\lambda}_{v}$ asymptotic to $\ell^{*}$. However, this is impossible: by Lemma 2.4 , the end of $\ell^{\prime}$ projects to a half-leaf in $S$ which is dense in $\lambda_{v}-$ but then $\ell^{*}$ would also project to a half-leaf which is dense in $\lambda_{v}$, implying that $\lambda_{v} \subset \lambda_{h}$. 
Thus, either $\left\{\ell_{i}^{*}\right\}$ or $\left\{\ell_{i}^{\prime}\right\}$ must leave every compact set. We take $L_{i}$ to be the corresponding leaves of $\widetilde{\Phi}_{h}$ or $\widetilde{\Phi}_{v}$.

For each $i$, let $H_{i}$ be the halfspace bounded by $L_{i} \times \mathbf{R}$ which contains the non-compact end of $\psi \circ \gamma^{\prime}$ (and therefore also of $\psi \circ \gamma$ ). Note that the sequence of halfspaces thus obtained is nested. Fixing a basepoint $x_{0} \in \widetilde{S}$, we see that $d_{|\tilde{\Phi}|}\left(x_{0}, L_{i}\right) \rightarrow \infty$, and eventually $\left(x_{0}, 0\right) \notin H_{i}$. We may therefore apply Lemma 7.3 to conclude that the diameters of $\tilde{f}\left(H_{i}\right)$ in the Poincare ball model of $\mathbf{H}^{3}$ converge to 0 , and therefore $\tilde{f}(\gamma)$ and $\tilde{f}\left(\gamma^{\prime}\right)$ have (the same) well-defined endpoint at infinity.

Thus we have shown that $F$ extends to a map $F: \mathbf{H}^{3} \cup \hat{\mathbf{C}} \rightarrow \mathbf{H}^{3} \cup \hat{\mathbf{C}}$, which is at least continuous when restricted to hyperbolic geodesic rays and their endpoints. We denote $\left.F\right|_{\hat{\mathbf{C}}}$ by $\pi$.

Continuity of the extension. Consider first continuity at a point $\xi \in \Lambda_{\Gamma_{0}}$. If $\gamma$ is a ray in $\mathbf{H}^{2}$ that terminates at this point, we construct, as in the proof of the existence of the map $\pi$, a sequence of halfspaces $H_{i}$ bounded by planes $L_{i} \times \mathbf{R}$, such that $\tilde{f}\left(H_{i}\right)$ converge to $F(\gamma(\infty))$ in the Poincare ball model. If $\xi_{n} \in \mathbf{H}^{3} \cup \hat{\mathbf{C}}$ is a sequence converging to $\xi$, let $\gamma_{n}:\left[0, T_{n}\right] \rightarrow \mathbf{H}^{3} \cup \hat{\mathbf{C}}$ be a sequence of geodesics based at some fixed $x \in \mathbf{H}^{3}$, such that $\gamma_{n}\left(T_{n}\right)=\xi_{n}$ (here we allow $T_{n}=\infty$ if $\left.\xi_{n} \in \hat{\mathbf{C}}\right)$. Since the halfspaces $\psi^{-1}\left(H_{i}\right)$ are quasiconvex in $\mathbf{H}^{3}$ and converging to $\xi$, it follows that for each $i$ there is an $n$ and a $t_{n}<T_{n}$ such that $\gamma_{n}\left(\left[t_{n}, T_{n}\right]\right) \subset \psi^{-1}\left(H_{i}\right)$. Therefore the images $F\left(\gamma_{n}\left(T_{n}\right)\right)$ converge to $F(\gamma(\infty))$. We conclude that $F$ and in particular its restriction $\pi$ is continuous at every point of $\Lambda_{\Gamma_{0}}$, which suffices to give Theorem $B$.

Now consider a point $\xi \in \Omega$. There is a unique $p \in \mathbf{H}^{2}$ and $\varepsilon= \pm 1$ such that the geodesic ray $\gamma(t)=(p, \varepsilon t)$ has endpoint $\gamma(\infty)=\xi$. Given $\xi_{n} \in \mathbf{H}^{3} \cup \hat{\mathbf{C}}$ converging to $\xi$, let $\gamma_{n}(t)=\left(p_{n}, \varepsilon t\right)$ and $T_{n} \in \mathbf{R} \cup\{\infty\}$ be such that $\gamma_{n}\left(T_{n}\right)=\xi_{n}$. We note that $p_{n} \rightarrow p$ and $T_{n} \rightarrow \infty$.

Recall the following fact: If $\left\{\alpha_{n}:\left[0, T_{n}\right) \rightarrow \mathbf{H}^{3}\right\}_{n=1}^{\infty}$ and $\alpha:[0, \infty) \rightarrow \mathbf{H}^{3}$ are $(K, \delta)$-quasigeodesics in $\mathbf{H}^{3}$ such that $d\left(\alpha_{n}(0), \alpha(0)\right)$ is bounded, then $\alpha_{n}\left(T_{n}\right) \rightarrow \alpha(\infty)$ if and only if there is a sequence $R_{n} \rightarrow \infty$ and a constant $C$, such that for all $n$ we have $d\left(\alpha_{n}(t), \alpha(t)\right) \leq C$ for $t \in\left[0, R_{n}\right]$ (see [Gro87, CDP90, GdlH90]).

There is therefore such a sequence $R_{n}$ and $C$ for the geodesics $\gamma_{n}$. By the analysis of Lemma 7.2, $F \circ \gamma_{n}$ and $F \circ \gamma$ are quasigeodesics (with uniform constants). Because $F$ is quasi-Lipschitz, there is a sequence $R_{n}^{\prime}$ and constant $C^{\prime}$ so that the same condition holds for $F \circ \gamma_{n}$ and $F \circ \gamma$. Thus $F\left(\xi_{n}\right)=$ $F\left(\gamma_{n}\left(T_{n}\right)\right)$ converge to $F(\xi)$.

The identifications of $\pi$. We conclude by showing that $\pi$ collapses $\hat{\mathbf{C}}$ in the manner claimed in the statement of the theorem. 
Let $\widetilde{\Phi}_{v}^{\infty} \equiv \psi^{-1}\left(\widetilde{\boldsymbol{\Phi}}_{v}\right) \times\{\infty\}$ denote the realization of the foliation $\psi^{-1}\left(\widetilde{\boldsymbol{\Phi}}_{v}\right)$ on the disk $\Omega_{+}$induced by our identification of $\mathbf{H}^{3}$ with $\mathbf{H}^{2} \times \mathbf{R}$ (extended to identify $\mathbf{H}^{3} \cup \Omega$ with $\left.\mathbf{H}^{2} \times[-\infty, \infty]\right)$. Similarly let $\widetilde{\boldsymbol{\Phi}}_{h}^{-\infty} \equiv \psi^{-1}\left(\widetilde{\boldsymbol{\Phi}}_{h}\right) \times\{\infty\}$ denote the realization of the horizontal foliation on $\Omega_{-}$.

We have already seen, in the discussion following Lemma 7.2, that $\pi$ maps the closure of every leaf of $\widetilde{\Phi}_{v}^{\infty}$ to a single point, and in the case of two degenerate ends maps the closure of every leaf of $\widetilde{\Phi}_{h}^{-\infty}$ to a single point. That is, each such leaf is obtained as a semicircle at infinity in one of the quasigeodesic planes $P=\psi^{-1}(L \times \mathbf{R})$, and we showed that $F$ extends on these planes to a map that collapses those semicircles to points, and is a homeomorphism on the rest.

It remains to show that there are no further identifications. This will follow from the following two lemmas:

Lemma 7.5. Given $q_{1}, q_{2} \in \widetilde{S}$ and $\varepsilon_{1}, \varepsilon_{2}= \pm 1$, let $\gamma_{i}(t)=\left(p_{i}, \varepsilon_{i} t\right) \in \widetilde{S} \times \mathbf{R}$ be vertical s-geodesic rays. If one of the following holds:

(1) $\varepsilon_{1}=\varepsilon_{2}=1$ and the $|\widetilde{\boldsymbol{\Phi}}|$-geodesic $\left[q_{1}, q_{2}\right]$ is not an arc of $\tilde{\boldsymbol{\Phi}}_{v}$,

(2) $\varepsilon_{1}=\varepsilon_{2}=-1$ and either $\left[q_{1}, q_{2}\right]$ is not an arc of $\widetilde{\Phi}_{h}$, or the metric $d s$ takes the form (5.8),

(3) $\varepsilon_{1}=-\varepsilon_{2}$,

then the rays $\gamma_{1}, \gamma_{2}$ diverge according to

$$
d_{s}\left(\gamma_{1}(t), \gamma_{2}(t)\right) \geq \min \left\{t, C e^{t / 2}\right\}
$$

for some $C=C\left(q_{1}, q_{2}\right)$.

Proof. We shall give the proof in case (1)-the other cases are similar. Thus, we may assume that $i\left(\left[q_{1}, q_{2}\right], \widetilde{\Phi}_{v}\right)=C>0$. Since $|\widetilde{\Phi}|$-geodesics minimize intersection number with $\widetilde{\Phi}_{h}$ and $\widetilde{\boldsymbol{\Phi}}_{v}$, we have $i\left(\alpha, \widetilde{\boldsymbol{\Phi}}_{v}\right) \geq C$ for all paths $\alpha$ joining $q_{1}$ to $q_{2}$. Furthermore, considering $|d x|$ as a form on all of $\tilde{S} \times \mathbf{R}$, we may conclude that $\int_{\alpha}|d x| \geq C$ for any $\alpha$ in $\tilde{S} \times \mathbf{R}$ whose endpoints are $\left(q_{i}, s_{i}\right)$ for any $s_{1}, s_{2} \in \mathbf{R}$.

Now for any given $t>0$, we observe that $\ell_{s}(\alpha) \geq C e^{t / 2}$ for any $\alpha$ connecting $\left(q_{1}, t\right)$ to $\left(q_{2}, t\right)$ which is contained in $\tilde{S} \times[t / 2, \infty)$. This comes directly from the following:

$$
\ell_{s}(\alpha)=\int_{\alpha}|d s| \geq \int_{\alpha} e^{\tau}|d x| \geq e^{t / 2} C
$$

(where $\tau$ is the component of $\alpha$ in the $\mathbf{R}$ direction). On the other hand if $\alpha$ is a path connecting $\left(q_{1}, t\right)$ to $\left(q_{2}, t\right)$ which exits $\widetilde{S} \times[t / 2, \infty)$, then $\ell_{s}(\alpha) \geq \int_{\alpha}|d \tau| \geq t$. The desired lower bound for $d_{s}\left(\gamma_{1}(t), \gamma_{2}(t)\right)$ follows.

A corollary of this lemma is the following:

Lemma 7.6. Let $L_{1}, L_{2}$ be leaves of $\widetilde{\Phi}_{h}$ or $\widetilde{\Phi}_{v}$ such that no full leaf of $\widetilde{\Phi}_{v}$ (nor, in the case of two degenerate ends, of $\widetilde{\Phi}_{h}$ ) intersects both of them. Then if 
$H_{i}($ for $i=1,2)$ are halfspaces of $\tilde{S} \times \mathbf{R}-\left(L_{i} \times \mathbf{R}\right)$ such that $H_{1} \cap H_{2}=\varnothing$, then the closed disks $D_{i}=\partial\left(\tilde{f}\left(H_{i}\right)\right)$ in $\hat{\mathbf{C}}$ are disjoint.

Proof. Let $\xi_{1}$ and $\xi_{2}$ be two points in $\partial D_{1}$ and $\partial D_{2}$, respectively. As we have seen, $\xi_{i}$ can be obtained as the endpoint $\tilde{f}\left(\gamma_{i}(\infty)\right)$ of a vertical geodesic $\gamma_{i}(t)=\left(q_{i}, \varepsilon_{i} t\right)$, where $q_{i} \in L_{i}$ and $\varepsilon_{i}= \pm 1$. The condition on $L_{1}$ and $L_{2}$ implies that $\gamma_{1}, \gamma_{2}$ satisfy one of the conditions of Lemma 7.5. Thus they diverge according to that lemma, and their images under the quasi-isometry $\tilde{f}$ cannot have the same endpoints. We conclude that $\partial D_{1} \cap \partial D_{2}=\varnothing$.

Thus, if $D_{1} \cap D_{2} \neq \varnothing$, one must be contained in the other-say $D_{1} \subset D_{2}$. But then, since $\tilde{f}\left(H_{i}\right)$ are quasiconvex in $\mathbf{H}^{3}$, we conclude that, for some $B$, $\widetilde{f}\left(H_{1}\right)$ lies in a $B$-neighborhood of $\tilde{f}\left(H_{2}\right)$. This contradicts the assumption that $H_{1} \cap H_{2}=\varnothing$.

Now let $\xi, \eta$ be two points in $\hat{\mathbf{C}}$ that are not supposed to be identified by $\pi$. There are several possibilities.

Suppose first that $\xi, \eta \in \Omega$. Then there are vertical rays $\gamma_{\xi}(t)=(p, \pm t)$ and $\gamma_{\eta}(t)=(q, \pm t)$ which terminate in $\xi$ and $\eta$, respectively. It will be sufficient to show that the rays $\psi \circ \gamma_{\xi}$ and $\psi \circ \gamma_{\eta}$ in $\tilde{S} \times \mathbf{R}$ diverge (in the $s$ metric), so that their images under $\widetilde{f}$ cannot have the same endpoint. Thus we apply Lemma 7.5. If $\xi, \eta \in \Omega_{+}$and they lie on different full leaves of $\widetilde{\Phi}_{v}^{\infty}$, we may apply part (1) of the lemma. If $\xi, \eta \in \Omega_{-}$and either $\Gamma$ has only one degenerate end or $\xi$ and $\eta$ lie on different full leaves of $\widetilde{\Phi}_{h}^{-\infty}$, we may apply part (2). Finally if $\xi \in \Omega_{+}$and $\eta \in \Omega_{-}$(or vice versa) we apply part (3).

Now consider the case when $\xi, \eta \in \Lambda_{\Gamma_{0}}$. We must show that $F(\xi) \neq F(\eta)$ if $\xi$ and $\eta$ are not endpoints of a full leaf of $\tilde{\Phi}_{v}^{\infty}$, or (in the case of two degenerate ends) of a full leaf of $\tilde{\Phi}_{h}^{-\infty}$. Consider the case of two degenerate ends-the proof in the other case is similar. Let $\left\{L_{i}\right\}_{i=-\infty}^{\infty}$ be a sequence of leaves of $\widetilde{\Phi}_{v}$ or $\widetilde{\Phi}_{h}$ such that $\psi^{-1}\left(L_{i}\right) \rightarrow \xi$ as $i \rightarrow \infty$ and $\psi^{-1}\left(L_{i}\right) \rightarrow \eta$ as $i \rightarrow-\infty$, as constructed in the proof of Lemma 7.4. Then there must be some $k$ for which there is no full leaf of either $\widetilde{\Phi}_{v}$ or $\widetilde{\Phi}_{h}$ which meets both $L_{k}$ and $L_{-k}$; for if not, we could take a limit and conclude that a full leaf of $\tilde{\Phi}_{v}^{\infty}$ or $\widetilde{\Phi}_{h}^{-\infty}$ connects $\xi$ and $\eta$, contradicting our assumption. Let $H_{k}$ and $H_{-k}$ be the halfspaces in the complements of $L_{k} \times \mathbf{R}$ and $L_{-k} \times \mathbf{R}$, respectively, which are disjoint. Then, as we have seen, $\pi(\xi) \in D_{k}$ and $\pi(\eta) \in D_{-k}$, where $D_{ \pm k}=\partial_{\infty}\left(\tilde{f}\left(H_{ \pm k}\right)\right)$. Thus the proof is completed by Lemma 7.6, which shows that $D_{k}$ and $D_{-k}$ are disjoint.

Finally, we must consider the possibility that $\xi \in \Lambda_{\Gamma_{0}}$ and $\eta \in \Omega$. However this case follows from the previous ones. For example if $\eta \in \Omega_{+}$then it lies on a full leaf $L$ of $\tilde{\Phi}_{v}^{\infty}$, but then $\pi(\eta)=\pi(\xi)$ if and only if $\xi$ lies in the closure of $L$, by appeal to the previous cases. 


\section{TOPOLOGICAL RIGIDITY FOR SURFACE GROUPS}

In this section we obtain an extension of Sullivan's rigidity theorem for the special case of surface groups satisfying a positive lower bound on injectivity radius.

Theorem E (Topological rigidity). Let $\Gamma_{1}$ and $\Gamma_{2}$ be two Kleinian groups, isomorphic to a closed surface group $\pi_{1}(S)$, whose quotients $\mathbf{H}^{3} / \Gamma_{i}$ satisfy $\mathrm{C} 2$. Suppose there is a homeomorphism $\mathscr{F}: \hat{\mathbf{C}} \rightarrow \hat{\mathbf{C}}$ such that

$$
\Gamma_{2}=\mathscr{F} \circ \Gamma_{1} \circ \mathscr{F}^{-1}
$$

(as groups acting on the sphere) and $\mathscr{F}$ is conformal on the domain of discontinuity of $\Gamma_{1}$. Then $\mathscr{F}$ is a Möbius transformation.

Remark. An alternate statement of this result is that two closed surface groups satisfying C2 which are topologically conjugate must also be quasiconformally conjugate (Sullivan's theorem then yields the rest). At any rate, for groups whose limit set is the whole sphere the conformality condition on the domain of discontinuity is vacuously satisfied.

Proof. Note first that the number of simply degenerate ends is the same in both groups, since $\mathscr{F}$ takes components of $\Omega_{\Gamma_{1}}$ to components of $\Omega_{\Gamma_{2}}$. If $\Gamma_{1}$ is quasi-Fuchsian then so is $\Gamma_{2}$, and in this case the theorem is already known (see [Mas70]). Thus we assume that at least one of the ends of $\Gamma_{1}$ is simply degenerate.

In view of Theorem $A$, we need to infer from the existence of the conjugating homeomorphism $\mathscr{F}$ that the groups $\Gamma_{1}$ and $\Gamma_{2}$ have identical ending laminations, and that $\mathscr{F}$ agrees with the conjugating map constructed in the proof of Theorem A. Let $G=\pi_{1}(S)$ and let $\rho_{i}: G \rightarrow \operatorname{PSL}_{2}(\mathrm{C})$ be representations such that $\Gamma_{i}=\rho_{i}(G)$ for $i=1,2$ and $\rho_{2}(g)=\mathscr{F} \rho_{1}(g) \mathscr{F}^{-1}$ for all $g \in G$. Choose a single Fuchsian representation $\rho_{0}$ of $G$ to be used in Theorem D for both groups.

Let $\pi_{1}, \pi_{2}: \hat{\mathbf{C}} \rightarrow \hat{\mathbf{C}}$ denote the collapsing maps given by Theorem $\mathrm{D}$ for $\Gamma_{1}, \Gamma_{2}$, respectively, and let $\widetilde{\Phi}_{ \pm}^{i}$ be the foliations on $\Omega_{ \pm}$along which $\pi_{i}$ collapse (if $\Gamma_{1}$ and $\Gamma_{2}$ have just one simply degenerate end, then we just have $\tilde{\Phi}_{+}^{i}$ on $\Omega_{+}$). To show that the foliations are identical we must first show that $\pi_{1}$ and $\pi_{2}$ collapse the same points on $S^{1}$, the limit set for $\rho_{0}(G)$.

Let $x, y \in S^{1}$ be such that $\pi_{1}(x)=\pi_{1}(y)$. Since $S^{1}$ is the limit set for $\rho_{0}(G)$, we can write $x=\lim _{i \rightarrow \infty} x_{i}$ and $y=\lim _{i \rightarrow \infty} y_{i}$, where $x_{i}$ are the attracting fixed points of some sequence $\left\{\rho_{0}\left(g_{i}\right)\right\} \subset \rho_{0}(G)$ and $y_{i}$ are the attracting fixed points of some sequence $\left\{\rho_{0}\left(h_{i}\right)\right\} \subset \rho_{0}(G)$. Because $\pi_{1}$ is continuous, we have

$$
\lim _{i \rightarrow \infty} \pi_{1}\left(x_{i}\right)=\lim _{i \rightarrow \infty} \pi_{1}\left(y_{i}\right)
$$


Further, it is clear that $\pi_{k}\left(x_{i}\right)$ are the attracting fixed points of $\rho_{k}\left(g_{i}\right)$, and likewise for $\pi_{k}\left(y_{i}\right)$ and $\rho_{k}\left(h_{i}\right)$, for $k=1,2$. Thus, since $\mathscr{F}$ conjugates $\rho_{1}$ to $\rho_{2}$, we have $\pi_{2}\left(x_{i}\right)=\mathscr{F}\left(\pi_{1}\left(x_{i}\right)\right)$ and similarly for $y_{i}$. It follows by continuity of $\mathscr{F}$ that

$$
\begin{aligned}
\lim _{i \rightarrow \infty} \pi_{2}\left(x_{i}\right) & =\lim _{i \rightarrow \infty} \mathscr{F}\left(\pi_{1}\left(x_{i}\right)\right) \\
& =\lim _{i \rightarrow \infty} \mathscr{F}\left(\pi_{1}\left(y_{i}\right)\right) \\
& =\lim _{i \rightarrow \infty} \pi_{2}\left(y_{i}\right),
\end{aligned}
$$

and therefore $\pi_{2}(x)=\pi_{2}(y)$. Interchanging $\pi_{1}$ with $\pi_{2}$ in this argument, and replacing $\mathscr{F}$ with $\mathscr{F}^{-1}$, we see that the opposite implication holds as well. Thus $\pi_{1}$ and $\pi_{2}$ identify the same points of $S^{1}$.

If $\Gamma_{1}$ and $\Gamma_{2}$ have just one degenerate end then we have shown that they have the same ending lamination. In this case we note also that, since $\mathscr{F}$ is assumed conformal on the domain of discontinuity of $\Gamma_{1}$, we may assume that $\rho_{0}$ is a Fuchsian representation uniformizing the resulting conformal structure on $S$. Thus we may assume that $\pi_{1}$ and $\pi_{2}$ are conformal on the lower hemisphere $\Omega_{-}$.

If $\Gamma_{1}$ and $\bar{\Gamma}_{2}$ have two degenerate ends then we have shown that every leaf of $\widetilde{\Phi}_{+}^{1}$ or $\widetilde{\Phi}_{-}^{1}$ occurs as a leaf of $\widetilde{\Phi}_{+}^{2}$ or $\widetilde{\Phi}_{-}^{2}$. But since each leaf of $\Phi_{h}^{i}$ on $S$ intersects every leaf of $\Phi_{v}^{i}$ and vice versa (each leaf is dense by Lemma 2.4), it follows that either $\Phi_{h}^{1}=\Phi_{h}^{2}$ and $\Phi_{v}^{1}=\Phi_{v}^{2}$, or vice versa, $\Phi_{h}^{1}=\Phi_{v}^{2}$ and $\Phi_{v}^{1}=\Phi_{h}^{2}$. We may assume without loss of generality that the first case occurs; the second case can be reduced to the first by precomposing $\pi_{2}$ with a reflection of $\hat{\mathbf{C}}$ through $S^{1}$.

Thus, since the ending foliations are the same, we may put the same model metric $s$ from Corollary 5.10 on $\widetilde{S} \times \mathbf{R}$ for both groups, and obtain both $\pi_{1}$ and $\pi_{2}$ as the extensions to $\hat{\mathbf{C}}$ of the maps $F_{i}=\tilde{f}_{i} \circ \psi$ constructed in the proof of Theorem D. Choosing a quasi-inverse $\tilde{f}_{1}^{-1}$ for $\tilde{f}_{1}$, we see that the quasi-isometry $\tilde{f}_{2} \circ \tilde{f}_{1}^{-1}$ extends to give the map $\mathscr{F}$ at infinity. As in Theorem A, we conclude that $\mathscr{F}$ is a Möbius transformation.

\section{APPENDIX: ENDING LAMINATIONS}

This appendix contains a more careful discussion of simply degenerate ends and ending laminations. These results are due to Thurston, but are not easily accessible in print. We also include here a discussion of the results from [Min93] which are the main new facts needed for the proof of Theorem A.

We shall assume throughout this section that $N$ is a hyperbolic manifold without cusps, satisfying Bonahon's condition $\mathrm{C} 1$. Recall from section 2.1 that an end of $N$ is simply degenerate if there is a sequence of simple closed curves on the corresponding boundary component of the compact core whose geodesic representatives exit the end. Thurston showed [Thu82a] that each of these 
geodesics can be completed to a lamination which can serve as the pleating locus for a pleated surface. Thus an equivalent definition of simple degeneracy is given by the following lemma. We note also that it is possible to replace the pleated surfaces by "simplicial hyperbolic surfaces" which are constructed with finite triangulations, as in [Bon86] and [Can93].

Lemma 9.1 (Thurston). An end $e$ of a manifold $N$ without cusps satisfying $\mathrm{C} 1$ is simply degenerate if and only if there is a sequence of pleated surfaces $\left\{g_{i}:\left(S_{e}, \rho_{i}\right) \rightarrow N\right\}$, homotopic to the inclusion map of $S_{e}$, which are eventually contained in any neighborhood of $e$.

We say that the pleated surfaces $g_{i}$ "exit the end".

Compactifications of $\mathscr{T}(S)$. The ending laminations can be understood heuristically as a limiting version of the Riemann surface invariants of geometrically finite ends, by recalling that $\mathscr{T}(S)$ can be compactified in several useful (non-equivalent) ways, so that the added points are identified with the sphere $\mathscr{P} \mathscr{M L}(S)$. Let us recall two such compactifications, Thurston's and Teichmüller's (see [Ker80, Mas82] for results on the similarities and differences between the two).

Thurston's compactification (see [Thua, FLP79]) uses the relation between intersection numbers of laminations and lengths in a degenerating sequence of metrics. In particular, as stated in Theorem 2.2 of [Thua] (see also [Thub] for the construction), a sequence $\left\{\rho_{i} \in \mathscr{T}(S)\right\}$ converges to $\beta \in \mathscr{P} \mathscr{M L}(S)$ if and only if there exists a sequence of measured laminations $\beta_{i}$ whose projective classes $\left[\beta_{i}\right]$ converge to $\beta$, such that for each $\mu \in \mathscr{M} \mathscr{L}\left(S_{e}\right)$ there is $C(\mu)$ such that for any $i$

$$
i\left(\mu, \beta_{i}\right) \leq \ell_{\rho_{i}}(\mu) \leq i\left(\mu, \beta_{i}\right)+C(\mu) .
$$

Furthermore, the lengths $\ell_{\rho_{1}}\left(\beta_{i}\right)$ go to infinity.

A Teichmüller compactification is obtained by fixing a basepoint $[\rho] \in \mathscr{T}(S)$ and considering the parametrization of $\mathscr{T}(S)$ by Teichmüller rays originating at $[\rho]$. To each ray we associate its endpoint, the vertical foliation of the quadratic differential, which determines a unique member of $\mathscr{P} \mathscr{M L}(S)$.

Using these compactifications, we can relate $\lambda_{e}$ to the asymptotics of the sequence of structures $\left\{\rho_{i}\right\}$ of the pleated surfaces in Lemma 9.1:

Lemma 9.2. Let $N$ be a hyperbolic 3-manifold without cusps, satisfying condition C1. Let $\left\{g_{i}:\left(S_{e}, \rho_{i}\right) \rightarrow N\right\}$ be a sequence of pleated surfaces exiting the end $e$. Let $L_{1}$ be the set of accumulation points of $\left\{\left[\rho_{i}\right]\right\}$ in Thurston's compactification of $\mathscr{T}(S)$. Fixing a basepoint for $\mathscr{T}(S)$, let $L_{2}$ be the set of accumulation points in the corresponding Teichmüller compactification. Then $L_{1}$ and $L_{2}$ consist of (projectivized) measured laminations with the same support, $\lambda_{e}$.

Note that we are not imposing condition $\mathrm{C} 2$ on $N$ in this theorem. This is because the proof of the general case is not much harder than the restricted case. However we still avoid the case of cusps, which involves further technicalities. 
Proof. As we will see in a moment, the sequence $\left\{\left[\rho_{i}\right]\right\}$ must leave every compact set in $\mathscr{T}\left(S_{e}\right)$, so that $L_{1}$ and $L_{2}$ are each contained in $\mathscr{P} \mathscr{L} \mathscr{L}\left(S_{e}\right)$. Consider $\beta \in \mathscr{M L}\left(S_{e}\right)$ representing a point in $L_{1}$, and assume (restricting to a subsequence if necessary) that $\left[\rho_{i}\right] \rightarrow[\beta]$ in Thurston's compactification. Let $\beta_{i} \in \mathscr{M L}(S)$ be a sequence such that $\left[\beta_{i}\right] \rightarrow[\beta]$ in $\mathscr{P} \mathscr{M L}\left(S_{e}\right)$, and so that the inequalities (9.1) hold relating intersection with $\beta_{i}$ to length in $\rho_{i}$.

Any geodesic lamination admits a non-zero measure (see [Thu82a, Theorem 8.10.6] or [Lev83]), although the measure may be supported in a sublamination. Choose such a measure for the pleating locus of $g_{i}$, denoting by $\lambda_{i}$ the resulting element of $\mathscr{M L}\left(S_{e}\right)$. Normalize in the $\rho_{1}$ metric via

$$
\hat{\lambda}_{i}=\frac{1}{\ell_{\rho_{1}}\left(\lambda_{i}\right)} \lambda_{i} .
$$

Then (taking a further subsequence if needed), we have $\hat{\lambda}_{i} \rightarrow \lambda \in \mathscr{M} \mathscr{L}\left(S_{e}\right)$ where, by Lemma $2.3, \operatorname{supp}(\lambda)=\lambda_{e}$.

Our task is to use these two sequences of laminations to show that $\operatorname{supp}(\lambda)=$ $\operatorname{supp}(\beta)$. We will use the fact that (see Lemmas 2.3 and 2.4) the lamination $\lambda_{e}$ intersects every other lamination on $S$, and consists of a single minimal component. It follows that, for any measured lamination $\alpha$,

$$
\operatorname{supp}(\alpha)=\lambda_{e} \quad \Longleftrightarrow \quad i(\alpha, \lambda)=0 .
$$

We show first that the normalized pleating laminations $\hat{\lambda}_{i}$ grow very short in $\rho_{i}$. If $d_{N}\left(g_{i}\left(S_{e}\right), g_{1}\left(S_{e}\right)\right)>D$, then

$$
\ell_{\rho_{i}}\left(\lambda_{i}\right) \leq(\cosh D)^{-1} \ell_{\rho_{1}}\left(\lambda_{i}\right)
$$

This follows just as in Lemma 4.3 if $\lambda_{i}$ is a simple closed curve. If $\lambda_{i}$ is not a closed curve, we can approximate it by simple closed curves whose geodesic representatives are as close as we like to $g_{i}\left(\lambda_{i}\right)$ and obtain the inequality in the limit. (We are using here the fact that the length of the realization of a lamination depends continuously on the lamination; see [Thua, Proposition 3.1] for a statement, and [Thu82a, CEG87] for exposition of the techniques needed.)

Thus, $\ell_{\rho_{i}}\left(\hat{\lambda}_{i}\right) \leq(\cosh D)^{-1} \rightarrow 0$. Note that it now clearly follows that $\left[\rho_{i}\right]$ leaves every compact set in $\mathscr{T}\left(S_{e}\right)$. Let $\hat{\beta}_{i}$ denote $\beta_{i}$ divided by its $\rho_{1}$-length, so that $\hat{\beta}_{i} \rightarrow\left(1 / \ell_{\rho_{1}}(\beta)\right) \beta$.

We now show $i\left(\hat{\beta}_{i}, \hat{\lambda}_{i}\right) \rightarrow 0$. Applying the first inequality of (9.1) to $\mu=\hat{\lambda}_{i}$ we conclude that

$$
i\left(\hat{\lambda}_{i}, \beta_{i}\right) \rightarrow 0 .
$$

Since $\ell_{\rho_{1}}\left(\beta_{i}\right) \rightarrow \infty$, even after normalizing $\beta_{i}$, we get $i\left(\hat{\lambda}_{i}, \hat{\beta}_{i}\right) \rightarrow 0$, so that

$$
i(\lambda, \beta)=0,
$$

and the lemma follows for $L_{1}$. 
The proof for $L_{2}$ is similar, but we must first recall the notion of extremal length for elements of $\mathscr{M} \mathscr{F}(S) \cong \mathscr{M} \mathscr{L}(S)$. Extremal length is an invariant of curves on a Riemann surface $(S, \sigma)$, invented by Ahlfors and Beurling (see [Ahl73]). Kerckhoff [Ker80] showed that it extends to a function $E_{\sigma}: \mathscr{M F}(S) \rightarrow \mathbf{R}_{+}$, which scales quadratically with the measure; that is,

$$
E_{\sigma}(c \lambda)=c^{2} E_{\sigma}(\lambda)
$$

We shall need to know the following relation of $E_{\sigma}$ to Teichmüller distance (also from [Ker80]):

$$
d_{T}(\rho, \sigma)=\sup _{\gamma \in \mathscr{K}(S)} \frac{1}{2} \log \frac{E_{\rho}(\gamma)}{E_{\sigma}(\gamma)}
$$

for any $[\rho],[\sigma] \in \mathscr{T}(S)$. In fact, the vertical foliation of the quadratic differential determining the Teichmüller geodesic from $[\rho]$ to $[\sigma]$ actually realizes this supremum. That is, if $\beta$ is the endpoint in $\mathscr{M F}(S)$ of the ray based at $[\rho]$ and passing through $\sigma$, then

$$
\frac{E_{\rho}(\beta)}{E_{\sigma}(\beta)}=\exp 2 d_{T}(\rho, \sigma) .
$$

The similarity of these facts to Lemma 2.5 is not accidental. There is the following relationship between extremal and hyperbolic lengths:

$$
c_{1} \ell_{\sigma}^{2}(\gamma) \leq E_{\sigma}(\gamma) \leq c_{2}(\sigma) \ell_{\sigma}^{2}(\gamma),
$$

where $c_{1}$ depends only on $\chi(S)$, and $c_{2}$ depends only on the minimum injectivity radius in the hyperbolic metric representing $[\sigma]$ (see [Mas85] as well as [Min93]).

We also need the following inequality:

$$
i\left(\alpha_{1}, \alpha_{2}\right)^{2} \leq E_{\sigma}\left(\alpha_{1}\right) E_{\sigma}\left(\alpha_{2}\right)
$$

for any $\alpha_{1}, \alpha_{2} \in \mathscr{M} \mathscr{L}(S)$ and $[\sigma] \in \mathscr{T}(S)$. This is an elementary fact; a proof can be found in [Min93], for example.

Now fix a basepoint $[\rho] \in \mathscr{T}\left(S_{e}\right)$, and let $\beta_{i} \in \mathscr{M} \mathscr{L}(S)$ represent the endpoint of the ray based at $[\rho]$ and passing through $\left[\rho_{i}\right]$. Then, possibly restricting to a subsequence, $\left[\beta_{i}\right]$ converges to $[\beta]$ in $L_{2}$. As in the first half of the proof we now bound $i\left(\beta_{i}, \lambda_{i}\right)$.

Since $d_{T}\left(\rho, \rho_{i}\right) \rightarrow \infty$, we conclude from (9.4) that $E_{\rho_{i}}\left(\beta_{i}\right) / E_{\rho}\left(\beta_{i}\right) \rightarrow 0$. Thus, normalize this time by

$$
\hat{\beta}_{i}=\frac{1}{\sqrt{E_{\rho}\left(\beta_{i}\right)}} \beta_{i},
$$

so that $\hat{\beta}_{i} \rightarrow E_{\rho}(\beta)^{-1 / 2} \beta$, and $E_{\rho_{i}}\left(\hat{\beta}_{i}\right) \rightarrow 0$.

Let us now adjust the sequence $\lambda_{i}$ so that the normalized sequence $\hat{\lambda}_{i}=$ $E_{\rho}\left(\lambda_{i}\right)^{-1 / 2} \lambda_{i}$ also has vanishing extremal lengths in $\rho_{i}$. Fix $\epsilon>0$ less than the 
Margulis constant for $\mathbf{H}^{3}$. If $g_{i}\left(S_{e}\right)$ misses the $\epsilon$-thin part of $N$ then there is a lower bound of $\epsilon$ on the injectivity radius in $\rho_{i}$, so that $E_{\rho_{i}}\left(\lambda_{i}\right) \sim \ell_{\rho_{i}}^{2}\left(\lambda_{i}\right)$, by (9.5). Suppose that this holds for infinitely many $g_{i}$. Then, after passing to a subsequence, we may conclude

$$
E_{\rho_{i}}\left(\hat{\lambda}_{i}\right)=\frac{E_{\rho_{i}}\left(\lambda_{i}\right)}{E_{\rho}\left(\lambda_{i}\right)} \rightarrow 0
$$

by virtue of (9.2). Applying (9.6) we obtain

$$
i\left(\hat{\beta}_{i}, \hat{\lambda}_{i}\right) \rightarrow 0 \text {, }
$$

so that $i(\beta, \lambda)=0$, concluding the proof in this case.

If, on the other hand, for infinitely many $i$, the image $g_{i}(S)$ passes through a component $T_{i}$ of the $\epsilon$-thin part of $N$ corresponding to some geodesic $\gamma_{i}$ in $N$, then (see [Thu86, proof of Proposition 5.9]) $g_{i}^{-1}\left(T_{i}\right)$ is contained in the $\epsilon^{\prime}$-thin part of $\left(S, \rho_{i}\right)$, where $\epsilon^{\prime}$ depends on $\epsilon$ and $\lim _{\epsilon \rightarrow 0} \epsilon^{\prime}=0$. In particular suppose that $\epsilon$ is sufficiently small that $\epsilon^{\prime}$ is also less than the Margulis constant. Then there is some simple closed curve $\lambda_{i}^{\prime}$ in $S_{e}$ whose image is homotopic to $\gamma_{i}$ and such that $\ell_{\rho_{i}}\left(\lambda_{i}^{\prime}\right) \leq \epsilon^{\prime}$. The sequence $\left\{\lambda_{i}^{\prime}\right\}$ must exit the end $e$ as well, since only finitely many thin parts $T_{i}$ can meet a compact subset of $N$. Thus, by Lemma $2.3,\left[\lambda_{i}^{\prime}\right] \rightarrow\left[\lambda^{\prime}\right] \in \mathscr{P} \mathscr{M L}\left(S_{e}\right)$ with $\operatorname{supp}\left(\lambda^{\prime}\right)=\lambda_{e}$.

Since $\ell_{\rho_{i}}\left(\lambda_{i}^{\prime}\right) \leq \epsilon^{\prime}$ we may conclude that $E_{\rho_{i}}\left(\lambda_{i}^{\prime}\right)$ is bounded, because for sufficiently short curves on arbitrary surfaces, $E$ is bounded above by a multiple of $\ell$ (see [Mas85]). On the other hand, $E_{\rho}\left(\lambda_{i}^{\prime}\right) \rightarrow \infty$, since there are only a finite number of curves of $\rho$-length less than any given constant. Normalizing as above, we have

$$
E_{\rho_{i}}\left(\hat{\lambda}_{i}^{\prime}\right) \rightarrow 0
$$

Again, by (9.6) it follows that

$$
i\left(\hat{\beta}_{i}, \hat{\lambda}_{i}^{\prime}\right) \rightarrow 0
$$

and therefore $i(\beta, \lambda)=0$.

Note. Suppose that, in the proof of the above theorem, we replace the metrics $\rho_{i}$ with hyperbolic metrics $\rho_{i}^{\prime}$ which differ by a bounded distortion. The relevant behavior of the extremal and hyperbolic lengths of curves in the proof (convergence to zero or $\infty$ ) would be unchanged by this perturbation, so the same conclusion would hold for the accumulation points of the $\rho_{i}^{\prime}$. Thus we may state:

Corollary 9.3. The conclusions of Lemma 9.2 hold if the metrics $\rho_{i}$ are replaced by $\rho_{i}^{\prime}$ such that $d_{T}\left(\rho_{i}, \rho_{i}^{\prime}\right)$ is uniformly bounded above.

Ends and Teichmüller rays. Lemma 9.2 shows that the invariant $\lambda_{e}$ gives some information about the geometry of $N$ in a neighborhood of the end $e$, by means of the sequence of metrics $\rho_{i}$ on the pleated surfaces in the end. This 
information is incomplete, however: it encodes only the asymptotics of the sequence, without further control on where in $\mathscr{T}\left(S_{e}\right)$ the points $\left[\rho_{i}\right]$ lie. The expectation is, at least for a manifold satisfying $\mathrm{Cl}-2$, that $\left[\rho_{i}\right]$ should lie in a bounded neighborhood of a geodesic. In fact, one can interpret Lemma 4.8 as the statement that, given conditions $\mathrm{Cl}-2$, the points $\left[\rho_{i}\right]$ describe a "quasigeodesic" in a certain sense. However, this property is not enough in general to imply that they lie near a true geodesic. Some use of the global geometry of Teichmüller space is needed to obtain this additional information, as well as the introduction of a further technique for controlling maps of surfaces into $N$, namely harmonic mappings. This is carried out in [Min92] and [Min93], where the following is shown:

Theorem 9.4 (Theorem A of [Min93]). Let $N$ satisfy C2. If $f: S \rightarrow N$ is an incompressible map, then there is a Teichmüller geodesic segment, ray, or line $L \subset \mathscr{T}(S)$ and a constant $A$ (depending on the $\epsilon$ in $C 2$ ) such that for every pleated surface $g:(S, \rho) \rightarrow N$ homotopic to $f$, the structure $[\rho]$ is at most a distance $A$ from $L$. Conversely, for each $[\sigma] \in L$ there is a pleated surface $g:(S, \rho) \rightarrow N$ homotopic to $f$, with $d(\sigma, \rho) \leq A$.

If $f$ is the inclusion map of a boundary component $S_{e} \subset \partial K(N)$ for a simply degenerate end e, then $L$ contains at least a Teichmüller ray.

We can now restate Theorem 5.5 (Ending ray), and show how it follows from Theorem 9.4.

Theorem 5.5 (Ending ray). Fix a compact 3-manifold $\bar{N}$ whose interior $N_{0}$ admits hyperbolic structures, and such that $\pi_{1}(\bar{N})$ is indecomposable (condition C1). For each boundary component $S_{e}$ of $\bar{N}$ (where $e$ denotes the corresponding end of $\left.N_{0}\right)$, fix a basepoint $\left[\sigma_{e}\right]$ in $\mathscr{T}\left(S_{e}\right)$.

If $N$ is a hyperbolic manifold homeomorphic to $N_{0}$ and satisfying $\mathrm{C} 2$, then each simply degenerate end $e$ of $N$ gives rise to a unique Teichmüller ray $R\left(\sigma_{e}, \Phi_{e}\right)$, which satisfies the conclusions of Theorem 9.4, and such that the vertical foliation of $\Phi_{e}$ is equivalent to the ending lamination $\lambda_{e}$.

Proof. Let $N$ satisfy $\mathrm{C} 1$ and $\mathrm{C} 2$, and let $S_{e}$ be the boundary component of $K(N)$ corresponding to a simply degenerate end $e$. Theorem 9.4 gives some ray $R=R(\sigma, \Phi)$ in $\mathscr{T}\left(S_{e}\right)$ such that any point on $R$ lies near the induced metric of a pleated surface homotopic to the inclusion. It remains only to observe that $\sigma$ and $\Phi$ are as claimed.

Corollary 9.3 of Lemma 9.2 implies that the measured foliation $\Phi_{e, v}$, which represents the unique accumulation point of $R$ in the Teichmüller compactification, must have underlying foliation which is equivalent to the ending lamination $\lambda_{e}$. To see that $R$ is unique (given $\sigma$ ), we must show that $\Phi$ is the unique quadratic differential with this property, up to a real scaling factor.

We say that a ray (or line) $R$ in $\mathscr{T}\left(S_{e}\right)$ is precompact if its projection to the moduli space $\mathscr{M}\left(S_{e}\right)$ lies in a compact subset. In other words, $R$ is precompact exactly if there is a positive lower bound to $\operatorname{inj}_{\sigma}$ for all $[\sigma] \in R$. 
A result of Masur [Mas, Theorem 1.1] says that if a ray $R(\sigma, \Phi)$ is precompact, then its endpoint $\Phi_{v}$ is uniquely ergodic. That is, the underlying foliation supports a unique transverse measure up to scaling, namely the one induced by $|\Phi|$.

In our case, the ray $R$ is clearly precompact, since the injectivity radius condition on $N$ implies the same lower bound on each pleated surface metric [ $\rho$ ] near any point of $R$. Thus $\Phi$ is uniquely determined by $\lambda_{e}$ (up to scaling).

Furthermore, the choice of the basepoint $[\sigma]$ is in fact irrelevant: By another theorem of Masur [Mas80], the unique ergodicity of $\Phi_{v}$ implies that if we choose a new ray $R\left(\sigma^{\prime}, \Phi^{\prime}\right)$ where $\Phi^{\prime}$ is the unique holomorphic quadratic differential in $\left(S, \sigma^{\prime}\right)$ such that $\Phi_{v}^{\prime}$ is measure-equivalent to $\Phi_{v}$, then this ray is asymptotic to the original one. Thus, the statement of Theorem 9.4 is still true for this ray, at the expense of changing the constants. It follows that we may assume that $\sigma$ is $\sigma_{e}$, the fixed basepoint of $\mathscr{T}\left(S_{e}\right)$.

\section{ACKNOWLEDGMENTS}

I would like to thank Francis Bonahon, Dick Canary, and Sergio Fenley for some very useful discussions, and for general encouragement. I am also grateful to the referees, who read the manuscript thoroughly and made many useful suggestions.

\section{REFERENCES}

[AB60] L. Ahlfors and L. Bers, Riemann's mapping theorem for variable metrics., Ann. of Math. (2) 72 (1960), 385-404.

[Abi80] W. Abikoff, The real-analytic theory of Teichmüller space, Lecture Notes in Math., vol. 820, Springer-Verlag, Berlin and New York, 1980.

[Abi88] Kleinian groups - geometrically finite and geometrically perverse, Geometry of Group Representations, Contemporary Math., no. 74, Amer. Math. Soc., Providence, RI, 1988, pp. 11-50.

[Ah173] L. Ahlfors, Conformal invariants: topics in geometric function theory, McGraw-Hill, New York, 1973.

[Bea83] A. F. Beardon, The geometry of discrete groups, Springer-Verlag, Berlin and New York, 1983.

[Ber60] L. Bers, Simultaneous uniformization, Bull. Amer. Math. Soc. 66 (1960), 94-97.

[Ber70] - Spaces of Kleinian groups, Maryland Conference in Several Complex Variables I, Lecture Notes in Math, vol. 155, Springer-Verlag, Berlin and New York, 1970, pp. 9-34.

[Bon] F. Bonahon, Bouts des variétés hyperboliques de dimension 3, prepublications de Orsay, 1985.

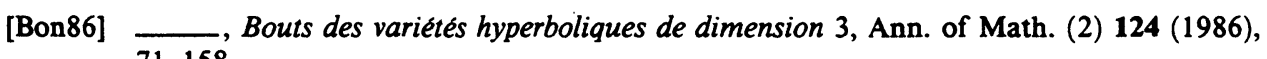
71-158.

[Can91] J. Cannon, The theory of negatively curved spaces and groups, Ergodic Theory, Symbolic Dynamics, and Hyperbolic Spaces (Trieste, 1989), Oxford Univ. Press, London and New York, 1991, pp. 315-369.

[Can93] R. D. Canary, Ends of hyperbolic 3-manifolds, J. Amer. Math. Soc. 6 (1993), 1-35.

[Cas82] A. J. Casson, Automorphisms of surfaces after Nielsen and Thurston, Notes by S. A. Bleiler, U. T. Austin, 1982.

[CB88] A. J. Casson and S. A. Bleiler, Automorphisms of surfaces after Nielsen and Thurston, Cambridge Univ. Press, London and New York, 1988. 
[CDP90] M. Coornaert, T. Delzant, and A. Papadopoulos, Géometrie et theorie de groupes: les groups hyperboliques de Gromov, Springer-Verlag, Berlin and New York, 1990.

[CEG87] R. D. Canary, D. B. A. Epstein, and P. Green, Notes on notes of Thurston, Analytical and Geometric Aspects of Hyperbolic Space, London Math. Soc. Lecture Note Ser., no. 111, Cambridge Univ. Press, Cambridge and New York, 1987, pp. 3-92.

[CT89] J. Cannon and W. Thurston, Group invariant peano curves, preprint, 1989.

[EM87] D. B. A. Epstein and A. Marden, Convex hulls in hyperbolic space, a theorem of Sullivan, and measured pleated surfaces, Analytical and Geometric Aspects of Hyperbolic Space, London Math. Soc. Lecture Note Ser., no. 111, Cambridge Univ. Press, Cambridge and New York, 1987, pp. 113-254.

[Fen92] S. Fenley, Asymptotic properties of depth one foliations in hyperbolic 3-manifolds, J. Differential Geom. 36 (1992), 269-313.

[Flo80] W. J. Floyd, Group completions and limit sets of Kleinian groups., Invent. Math. 57 (1980), 205-218.

[FLP79] A. Fathi, F. Laudenbach, and V. Poenaru, Travaux de Thurston sur les surfaces, vol. 66-67, Asterisque, 1979.

[Gar87] F. Gardiner, Teichmüller theory and quadratic differentials, Wiley Interscience, New York, 1987.

[GdlH90] E. Ghys and P. de la Harpe (eds.), Sur les groupes hyperboliques d'aprés Mikhael Gromov, Birkhaüser, Basel, 1990.

[GM91] F. Gardiner and H. Masur, Extremal length geometry of Teichmüller space, Complex Variables Theory Appl. 16 (1991), 209-237.

[Gro87] M. Gromov, Hyperbolic groups, Essays in Group Theory (S. M. Gersten, ed.), Math. Sci. Res. Inst. Publ., no. 8, Springer-Verlag, Berlin and New York, 1987.

[Hat88] A. E. Hatcher, Measured lamination spaces for surfaces, from the topological viewpoint, Topology Appl. 30 (1988), 63-88.

[HM79] J. Hubbard and H. Masur, Quadratic differentials and foliations, Acta Math. 142 (i979), 221-274.

[Ker80] S. Kerckhoff, The asymptotic geometry of Teichmüller space, Topology 19 (1980), 23-41.

[Ker92] _ Lines of minima in Teichmüller space, Duke Math. J. 65 (1992), 187-213.

[Kra72] I. Kra, On spaces of Kleinian groups, Comment. Math. Helv. 47 (1972), 53-69.

[Lev83] G. Levitt, Foliations and laminations on hyperbolic surfaces, Topology 22 (1983), 119135.

[Mar74] A. Marden, The geometry of finitely generated Kleinian groups, Ann. of Math. (2) 99 (1974), 383-462.

[Mas] H. Masur, Hausdorff dimension of the set of nonergodic foliations of a quadratic differential, preprint.

[Mas70] B. Maskit, On boundaries of Teichmüller spaces and on Kleinian groups II, Ann. of Math. (2) 91 (1970), 607-639.

[Mas71] - Self-maps of Kleinian groups, Amer. J. Math. 93 (1971), 840-856.

[Mas80] H. Masur, Uniquely ergodic quadratic differentials, Comment. Math. Helv. 55 (1980), 255-266.

[Mas82] _ , Two boundaries of Teichmüller space, Duke Math. J. 49 (1982), 183-190.

[Mas85] B. Maskit, Comparison of hyperbolic and extremal lengths, Ann. Acad. Sci. Fenn. 10 (1985), 381-386.

[McM89] C. McMullen, Amenability, Poincaré series and quasiconformal maps, Invent. Math. 97 (1989), 95-127.

[Min92] Y. Minsky, Harmonic maps into hyperbolic 3-manifolds, Trans. Amer. Math. Soc. 332 (1992), 607-632.

[Min93] , Teichmüller geodesics and ends of hyperbolic 3-manifolds, Topology 32 (1993), 625-647. 
[Mos68] G. D. Mostow, Quasiconformal mappings in n-space and the rigidity of hyperbolic space forms, Inst. Hautes Études Sci. Publ. Math. 34 (1968), 53-104.

[Mos73] _ Strong rigidity of locally symmetric spaces, Ann. of Math. Stud., no. 78, Princeton Univ. Press, Princeton, NJ, 1973.

[Ohs] K. Ohshika, Topologically conjugate Kleinian groups, preprint.

[Pra73] G. Prasad, Strong rigidity of Q -rank 1 lattices, Invent. Math. 21 (1973), 255-286.

[Sco73] P. Scott, Compact submanifolds of 3-manifolds, J. London Math. Soc. 7 (1973), 246-250.

[Str84] K. Strebel, Quadratic differentials, Springer-Verlag, Berlin and New York, 1984.

[Sul81] D. Sullivan, On the ergodic theory at infinity of an arbitrary discrete group of hyperbolic motions, Riemann Surfaces and Related Topics: Proceedings of the 1978 Stony Brook Conference, Ann. of Math. Stud., no. 97, Princeton Univ. Press, Princeton, NJ, 1981.

[Thua] W. Thurston, Hyperbolic structures on 3-manifolds, II: surface groups and manifolds which fiber over the circle, preprint.

[Thub] — Minimal stretch maps between hyperbolic surfaces, unpublished manuscript, Princeton University.

[Thu82a] _ The geometry and topology of 3-manifolds, Princeton University lecture notes, 1982.

[Thu82b] _ Three dimensional manifolds, Kleinian groups and hyperbolic geometry, Bull. Amer. Math. Soc. (N.S.) 6 (1982), 357-381.

[Thu86] — Hyperbolic structures on 3-manifolds, I: deformation of acylindrical manifolds, Ann. of Math. (2) 124 (1986), 203-246.

\begin{abstract}
Thurston's ending lamination conjecture states that a hyperbolic manifold is uniquely determined by a collection of Riemann surfaces and geodesic laminations that describe the asymptotic geometry of its ends. We prove this conjecture for the case of manifolds whose fundamental group is freely indecomposable, and which admit a positive lower bound on injectivity radii.

The techniques of the proof apply to show that a Kleinian surface group admitting a positive lower bound on injectivity radii is continuously semiconjugate to a Fuchsian group. This extends results of Cannon and Thurston.

A further consequence is a rigidity theorem for surface groups satisfying the injectivity radius condition, namely that two such groups whose actions on the sphere are conjugate by a homeomorphism that is conformal on the domains of discontinuity must be conjugate by a Möbius transformation.
\end{abstract}

Department of Mathematics, State University of New York at Stony Brook, Stony BROOK, NEW YORK 11794

E-mail address: yair@math. sunysb.edu 